\title{
Translation Symmetry Breaking in Four Dimensional Lattice Gauge Theories
}

\section{Christian Borgs}

Max-Planck-Institut für Physik und Astrophysik, Werner-Heisenberg-Institut für Physik, D-8000 München, Federal Republic of Germany

\begin{abstract}
We consider lattice gauge theories with finite abelian group $G$ in the weak coupling regime. It is shown that there is only one translation invariant equilibrium state for the infinite system. In four dimensions we construct a nontranslation invariant equilibrium state, describing an infinite system with localized magnetic flux tube, starting and ending at infinity.
\end{abstract}

\section{Introduction}

It has been expected for a while (and was independently proven by Aizenman [1] and Higuchi [2]) that in two dimensions the only possible equilibrium states for the Ising model are convex combinations of the two states $\mu_{+}, \mu_{-}$, which are the thermodynamic limit of the Gibbs states in finite volume with positive respectively negative boundary conditions (b.c.). This excludes the possibility of phase coexistence and breaking of translation invariance for the two dimensional Ising system.

In three and more dimensions however such phenomena can occur at sufficiently low temperatures. This was first shown by Dobrushin in [3], who induced such a state by mixed b.c. on increasing lattices: - on the lower and + on the upper halves of the surface of the lattice. In the resulting infinite volume state, the average magnetization will be near +1 in the upper and near -1 in the lower half of the system. A considerable simplification of the proof is due to van Beijeren [4].

For lattice gauge theories, at least to my knowledge, the possibility that the corresponding states might exist and describe translation invariance breaking has not been considered in the literature. In [5], for example, it is suggested that in the region of convergence of cluster expansions the thermodynamic limit is independent of b.c. This is true in the strong coupling regime of lattice gauge theories, but for the weak coupling regime considered in this paper it is at best true for some restricted class of b.c. 
It is clear that a phenomenon of translation invariance breaking in lattice gauge theories will not be accompanied by phase coexistence: such phases should, at least locally and far away from the corresponding "interface," be translation invariant. By a straightforward generalization of arguments presented in [6] we show in Sect. 2 that for fixed value of the coupling there is only one translation invariant Gibbs state in the infinite volume (provided we are in the weak coupling regime).

But the interface between two regions of opposite average magnetization in the Ising system has its counterpart for the gauge systems considered here: the objects dual to frustrated plaquettes. Due to the Bianchi-identity they form closed lines ("vortices") in $d=3$ and closed surfaces ("vortex-sheets") in $d=4$. And just like Peierls contours they can begin and end at the boundary.

So the problem studied in this paper can be formulated as follows:

1. Can we choose b.c. such that a vortex or vortex sheet is forced into the system?

2. Will such a vortex (sheet) be sharp, or will it fluctuate so much that it doesn't influence expectation values?

Whereas 1 . can be done for $d \geqq 3$, we expect that the vortex sheets will only be sharp for $d \geqq 4$. Note that for $d=3$ they form lines and in analog to the spin system we expect that they will fluctuate too much.

The organization of this paper is as follows: in Sect. 1 we introduce our notation and in particular introduce the analog to the Peierls contours, the defect network. An important notion will be that of global or exterior defect networks, which are defect networks (co)connecting to the boundary. In Sect. 2 we show uniqueness of the translation invariant states, using methods similar to those presented in [6]. In Sect. 3 we analyze the probabilities of the exterior defect networks and in Sect. 4 the conditional expectation values with fixed exterior defect network. The results are technically very important for Sect. 2 and Sect. 5 as well, but the reader not interested in technical detail could skip them, except perhaps the remarks after Lemmas 3.3 and 4.2, which summarize the main results needed in the sequel. The b.c. forcing a vortex sheet (i.e., a magnetic flux) into the systems are introduced in Sect. 5, where we also formulate the main theorem (which states that the corresponding equilibrium state is not translation invariant). With the preparations done in Sect. 3 and 4 the proof reduces essentially to the geometric study of the surface dual to the exerior defect network. Apart from some remarks concerning the general strategy - which are made after Lemma 5.3 - this is done in Sect. 6. Most of the ideas used in this section - including a "renormalization trick" - can be found already in [3]. The adaption to our situation is however not quite trivial, first because we are working in four dimensions instead of three, and second because vortices can split (if the gauge group is not $Z_{2}$ ) which is not the case for Peierls contours. I have tried to be as clear as possible (within the restrictions due to my own capability), but I fear that the reading of Sect. 6 still might cost a good deal of hard toil - so did the writing. Finally in Sect. 7 I summarize the results of this paper and discuss possible generalizations. In the appendix I present a corrected version of the proofs in [5] concerning cluster expansion convergence which is needed in Sects. 3 and 4. 


\section{Gauge Fields and Defect Networks}

We consider pure Yang-Mills theories in a box $\Lambda \subset \mathbb{Z}^{d}(d \geqq 3)$ with finite abelian gauge group $G$. Pick a positive orientation for the links and plaquettes of $\Lambda$ and denote the set of positively orientated links respectively plaquettes by $\Lambda_{t}$ respectively $\Lambda_{P}$. A gauge field configuration $g$ is a map from $\Lambda_{\ell}$ into $G:\langle x y\rangle \mapsto g_{x y}$. If $\langle y x\rangle \in \Lambda_{\ell}$ we define $g_{x y} \equiv g_{y x}^{-1}$. For each loop $C$ of links one defines the loop variable $g_{C}$ by multiplying the group elements $g_{x y}$ corresponding to links in $C$. In particular the plaquettes $P \in \Lambda_{P}$ will be mapped into group elements $g_{\partial P}$ which often will be denoted simply $g_{P}$.

To define the action $S$ of the model we assign to each plaquette $P \in \Lambda_{P}$ a realvalued, even function $s_{P}(\cdot)$ over $G$ (i.e. $s_{P}(g)=s_{P}\left(g^{-1}\right)$ is real for all $g$ in $G$ ) with unique minimum at $g=\mathbb{1}$, without loss $s_{P}(\mathbb{1})=0$. In the sequel we assume that $s_{P}$ does not depend on $P$, but all results of this paper remain valid, if $s_{P}$ depends on $P$ in a translation invariant fashion, i.e. $S_{P}=s_{P^{\prime}}$ if $P^{\prime}$ is a translate of $P$, which is relevant for example for the Hamiltonian limit. So we have

$$
S_{\Lambda}(g)=\sum_{P \in \Lambda_{P}} s\left(g_{\partial P}\right)
$$

where $s(\cdot)$ is a real-valued even function with

$$
s(g) \geqq 0 \text { for all } g \in G
$$

and

$$
s(g)=0 \quad \text { only for } \quad g=\mathbb{1} .
$$

For instance for the lattice gauge theory of Wegner [7] and Wilson [8]

$$
s_{W}(\cdot)=J \operatorname{Re}(\chi(\mathbb{1})-\chi(\cdot)),
$$

where the inverse coupling $J=2 / g^{2}$ is a real number $J \geqq 0$ and $\chi$ is a faithful character of $G$. For us it is only important that $s(\cdot)$ depends on $J$ in such a way that

$$
e^{-b} \equiv \sum_{g \neq \mathbb{1}} e^{-s(g)}
$$

goes to zero as $J \rightarrow \infty$.

Clearly this implies the same for

$$
e^{-b_{0}} \equiv \max _{g \neq \mathbb{1}} e^{-s(g)} .
$$

We finally recall the definition of the partition function and of expectation values. For the rest of this section we consider fixed boundary conditions (b.c.) $b$ on $\partial \Lambda$ :

$$
g_{x y}=b_{x y} \quad \text { if } \quad x, y \in \partial \Lambda .
$$

Then the partition function $Z_{\underline{b}}$ becomes

$$
Z_{b}^{A} \equiv \sum_{g}^{\prime} e^{-S_{\Lambda}(g)},
$$


and the expectation value of an observable $A$ (i.e. a bounded function $A$ of the gauge field configurations)

$$
\langle A\rangle_{\underline{b}}^{\Lambda} \equiv\left(Z_{\underline{b}}^{\wedge}\right)^{-1} \sum_{g}^{\prime} A(g) e^{-S_{\Lambda}(g)},
$$

where the sum in (1.7) and (1.8) goes over all gauge field configurations satisfying (1.6).

Without loss (see e.g. [9]) we can restrict ourselves to gauge invariant observables, i.e. observables with $A(\underset{g}{g})=A(\underbrace{\prime})$ if $g^{\prime}$ is obtained from $g$ by a gauge transformation (which means that $\widetilde{g_{x y}^{\prime}}=\dot{h}_{x} \tilde{g}_{x y} h_{y}^{-1}$ with some function $h$ from $A$ into $G)$.

We now define following [5]

Definition 1.1. A defect network $D$ is a function from $\Lambda_{P}$ into $G, P \mapsto g_{P}^{D}$. We write $P \in D$ if $P$ lies in the support of $D$, i.e. $g_{P}^{D} \neq \mathbb{1}$, and $|D|$ will denote the number of plaquettes $P \in D$. A defect network $D$ is allowed if it can be obtained from a gauge field configuration.

The following two Lemmas express the fact that instead of summing over gauge field configurations in (1.7) and (1.8) one can equivalently sum over allowed defect networks. The first Lemma is well known.

Lemma 1.2. An allowed defect network $D$ satisfies the Bianchi-identity

$$
\prod_{P \in \partial c} g_{P}^{D}=\mathbb{1}
$$

for all cubes $c$ in $\Lambda$.

Proof. By explicit calculation.

Lemma 1.3. i) A defect network $D$ is allowed if it satisfies the Bianchi-identity and is compatible with the b.c., i.e.

$$
g_{P}^{D}=\prod_{\langle x y\rangle \in \partial P} b_{x y}
$$

for plaquettes whose corners lie all in $\partial \Lambda$.

ii) The corresponding gauge field configuration is unique up to gauge transformations.

Proof. The proof is done by explicit construction. We first fix the gauge as follows: all link variables for links in time direction are set to one, except those corresponding to links in the last time slice (and clearly those which are already fixed by the b.c.). It is now an easy exercise to construct the gauge field configuration from the known values on the boundary and the plaquette variables. The Bianchi-identity ensures that we cannot run into contradictions. This proves i) and ii) at the same time.

Lemmas 1.2 and 1.3 now allow us to rewrite (1.8). First due to Lemma 1.3 we can write for gauge invariant observables and allowed defect networks $D$,

$$
A(D) \equiv A(\underset{\sim}{g})
$$


where $g$ is chosen such that

$$
g_{P}=D(P) \text { for all } P \in \Lambda_{P} \text {. }
$$

If we introduce the activity $z(D)$ of a defect network $D$ by

$$
z(D)=\prod_{P \in \Lambda_{P}} e^{-s(D(P))}
$$

we get

$$
\begin{gathered}
\langle A\rangle_{\underline{b}}^{A}=\left(\tilde{Z}_{\underline{b}}^{\Lambda}\right)^{-1} \sum_{D}^{\prime} z(D) A(D), \\
\tilde{Z}_{\underline{b}}^{A}=\sum_{D}^{\prime} z(D),
\end{gathered}
$$

where $\Sigma^{\prime}$ goes over defect networks, which are compatible with the b.c. and satisfy the Bianchi-identity.

In the sequel it is convenient to decompose a given defect network into a socalled interior and exterior one. Call two plaquettes coconnected, if there is an elementary cube $c$, such that both plaquettes lie in $c$.

Definition 1.4. An interior defect network is a defect network for which $D(P)=\mathbb{1}$ for all plaquettes in the boundary. A defect network is called an exterior defect network if its support decomposes into coconnectivity components, which all contain at least one plaquette in the boundary. Two defect networks $D, D^{\prime}$ are called compatible if for any plaquette $P \in D$ and $P^{\prime} \in D^{\prime} P$ and $P^{\prime}$ are not coconnected.

With these definitions it is evident that instead of summing over all allowed defect networks we can first sum over exterior defect networks $E$, satisfying the Bianchiidentity (denote this sum by $\sum$ ) and then over all interior ones satisfying the Bianchiidentity and being compatible with $E$ (denote this sum by $\Sigma^{\prime \prime}$ ):

$$
\langle A\rangle_{\underline{b}}^{\Lambda}=\left(\tilde{Z}_{\underline{b}}^{\Lambda}\right)^{-1} \sum_{E}^{\prime} z(E) \sum_{D}^{\prime \prime} z(D) A(E, D),
$$

where $A(E, D)$ is the same as $A\left(D^{\prime}\right)$ according to (1.9) with $D^{\prime}(P)=D(P) D(P)$. We rewrite the above equation a little bit

$$
\langle A\rangle_{\underset{\sim}{b}}^{A}=\sum_{E}^{\prime} p_{\underset{\sim}{b}}(E)\langle A\rangle_{E}^{A}
$$

with

$$
\begin{gathered}
p_{\underline{b}}(E) \equiv z(E) Z_{E} / \tilde{Z}_{\underline{b}}^{\Lambda}, \\
\tilde{Z}_{b}^{A}=\sum_{E}^{\prime} z(E) Z_{E}, \\
Z_{E} \equiv \sum_{D}^{\prime \prime} z(D), \\
\langle A\rangle_{E}^{A} \equiv Z_{E}^{-1} \sum_{D}^{\prime \prime} z(D) A(E, D) .
\end{gathered}
$$

Equation (1.13) will be the starting point for all the results presented in this paper. The probabilities $p_{b}(E)$ as well as the expectation values $\langle\cdot\rangle_{E}^{A}$ will therefore be studied in separate sections, Sects. 3 and 4 . In the sequel we often encounter a combinatoric constant $K_{0}$, which appears in the following 
Lemma 1.5. Let $P_{0}$ be a fixed plaquette in $\mathbb{Z}^{d}$. The number of coconnected sets $T$ of plaquettes in $\mathbb{Z}^{d}$ with fixed size $|T|=$ s, such that one of the plaquettes in $T$ coconnects to $P_{0}$ is bounded by $K_{0}^{s}$.

The proof is a well known consequence of the solution of the Königsberg bridge problem and can be found e.g. in [5].

\section{The Translation Invariant Equilibrium States}

In this section we show that for $J$ large enough there is only one translation invariant equilibrium state. In order to make the statement precise we introduce some definitions.

Consider the infinite system defined on $\mathbb{Z}^{d}$. A local observable $A$ is a bounded function from the gauge field configuration on $\mathbb{Z}^{d}$ into $\mathbb{C}$, which depends only on finitely many link variables. The corresponding set of links is called (with a slight abuse of notation) the support $\operatorname{supp}(A)$ of $A$. For a finite volume $\Lambda \subset \mathbb{Z}^{d}$ a finite volume state $\langle\cdot\rangle_{A}$ is a linear functional on the set of local observables with support in $\Lambda$, satisfying the normalization condition $\langle 1\rangle_{\Lambda}=1$. Following [10] an infinite volume state $\langle\cdot\rangle$ is given by a system $\left\{\langle\cdot\rangle_{\Lambda}\right\}_{\Lambda \subset \mathbb{Z}^{d}}$ satisfying the compatibility condition

$$
\langle A\rangle_{\Lambda}=\langle A\rangle_{\Lambda^{\prime}}
$$

if $\operatorname{supp}(A)$ is as well in $\Lambda$ as in $\Lambda^{\prime}$. With (2.1) we can define

$$
\langle A\rangle \equiv\langle A\rangle_{A},
$$

where $\Lambda$ has to be chosen so large that $\operatorname{supp}(A)$ lies in $\Lambda$.

Next we call the functions $s(\cdot)$ of the last section "interaction" and following Dobrushin, Lanford and Ruelle $[10,11]$ an infinite volume state $\langle\cdot\rangle$, given by the system $\left\{\langle\cdot\rangle_{A}\right\}$, is called equilibrium state for the interaction $s(\cdot)$ if the finite volume states $\langle\cdot\rangle_{A}$ can be written

$$
\langle\cdot\rangle_{\Lambda}=\int d \mu_{\Lambda}(\underline{b})\langle\cdot\rangle_{\underline{b}}^{A}
$$

with some probability measure $d \mu_{\Lambda}(\cdot)$ and $\langle\cdot\rangle_{b}^{\Lambda}$ according to (1.8).

Finally a state $\langle\cdot\rangle$ is called translation invariant if for any vector $x \in \mathbb{Z}^{d}$

$$
\langle A\rangle=\left\langle t_{x}(A)\right\rangle
$$

where $t_{x}(A)$ denotes the translate of $A$ by $x$.

We are now ready to state

Theorem 2.1. Let $J$ be so large that $b>\log K_{0}+\log 2.93$. Then the only translation invariant equilibrium state for the interaction $s(\cdot)$ is the state $\langle\cdot\rangle_{\mathbb{1}}$, obtained as thermodynamic limit from the states $\langle\cdot\rangle_{\mathbb{1}}^{\Lambda}$ with b.c. $\underset{\sim}{b}=\mathbb{1}$ (i.e. $b_{x y}=\mathbb{1}$, whenever $x, y \in \partial \Lambda)$.

Remarks. i) The existence of the thermodynamic limit for the b.c. $\underset{\sim}{b}=\mathbb{1}$ is shown in Sect. 4 [see Eq. (4.8)].

ii) The proof of the theorem can to a large extent be taken over from [6], but for the benefit of the reader we give the complete argument here. 
iii) For the properties of the state $\langle\cdot\rangle_{\mathbb{1}}$, like deconfinement for electric charges, confinement for magnetic monopoles in $d=4$, and exponential clustering for truncated expectation values we refer to Theorem 3.17 of [5]; note however that the proof of this theorem breaks down for general (nontranslation invariant) equilibrium states.

To prove Theorem 2.1 it is convenient to introduce averaged expectation values,

$$
\begin{aligned}
\langle\bar{A}\rangle_{\underline{b}}^{A} & \equiv \frac{1}{\left|\Lambda^{\prime}\right|} \sum_{x \in \Lambda^{\prime}}\left\langle t_{x}(A)\right\rangle_{\underline{\sim}}^{\Lambda}, \\
\langle\bar{A}\rangle_{\Lambda} & \equiv \frac{1}{\left|\Lambda^{\prime}\right|} \sum_{x \in \Lambda^{\prime}}\left\langle t_{x}(A)\right\rangle_{\Lambda},
\end{aligned}
$$

where $\Lambda^{\prime} \subset \mathbb{Z}^{d}$ is the set of points $x$ such that the support of $t_{x}(A)$ lies in $\Lambda$. Clearly we assumed that $\operatorname{supp}(A)$ lies in $\Lambda$. Due to (2.2) and (2.4) it is clear that for translation invariant equilibrium states

$$
\langle A\rangle=\langle\bar{A}\rangle_{A}
$$

if only $\operatorname{supp}(A)$ lies in $A$. With (2.3) Theorem 2.1 follows from

Lemma 2.2. Let $b>\log K_{0}+\log 2.93$. Then

$$
\left|\langle\bar{A}\rangle_{\underline{b}}^{\Lambda}-\langle A\rangle_{\mathbb{1}}\right| \leqq g(A, \Lambda),
$$

where $g(A, \Lambda)$ is a function independent of the b.c. and goes to zero if $\Lambda \rightarrow \mathbb{Z}^{d}$ through a sequence of hypercubes.

Proof of Theorem 2.1, using Lemma 2.2. Let $\langle\cdot\rangle$ be a translation invariant equilibrium state, $A$ a local observable. We have to show that $\langle A\rangle=\langle A\rangle_{\mathbb{1}}$. Equations (2.3), (2.7), and Lemma 2.2 yield

$$
\left|\langle A\rangle-\langle A\rangle_{\mathbb{1}}\right|=\left|\langle\bar{A}\rangle_{\Lambda}-\langle A\rangle_{\mathbb{1}}\right| \leqq g(A, \Lambda)
$$

if $\operatorname{supp} A$ lies in $\Lambda$. The right-hand side can be made arbitrarily small by choosing suitable $\Lambda$ 's. This proves that $\langle A\rangle=\langle A\rangle_{\mathbb{1}}$.

The proof of Lemma 2.2 is based on the following idea: the exterior defect network $E$ (see Sect. 1) has possibly a length of $|E| \sim|\partial \Lambda|$, since $E$ coconnects to the boundary. But it will be not very much larger, because large defect networks have small activity. Therefore for most of the points $x \in \Lambda^{\prime}$ the translates $t_{x}(A)$ will have support far away from $E$. But for such $x$ the expectation value $\left\langle t_{x}(A)\right\rangle_{E}$ will be nearly the same as $\left\langle t_{x}(A)\right\rangle_{\mathbb{1}}=\langle A\rangle_{\mathbb{1}}$.

The precise argument is based on the following two lemmas, which are proven in Sects. 3 and 4.

Lemma 2.3. Suppose $b>\log K_{0}$ and let $\varrho>0$. Then the probability that $E$ has size greater than $|\partial \Lambda|^{1+\varrho}$ goes to zero with $|\partial \Lambda| \rightarrow \infty$ uniformly in the b.c., i.e.

$$
\sum_{E:|E| \geqq|\partial \Lambda|^{1+\varrho}}^{\prime} p_{b}(E) \leqq \varepsilon(|\partial \Lambda|, \varrho),
$$

where $\varepsilon(|\partial \Lambda|, \varrho)$ is independent of the b.c. and for all $\varrho>0$ goes to 0 with $|\partial \Lambda| \rightarrow \infty$. $\Sigma^{\prime}$ is defined as in (1.13). 
Lemma 2.4. Let $b>\log K_{0}+\log 2.93$. Then

$$
\left|\langle A\rangle_{E}^{\Lambda}-\langle A\rangle_{\mathbb{1}}\right| \leqq \tilde{\varepsilon}\left(A, \min \left(d_{1}, d_{2}\right)\right),
$$

where $\tilde{\varepsilon}(A, d)$ depends in a translation invariant way on $A$ and goes to 0 if $d \rightarrow \infty . d_{1}$ is the distance from $\operatorname{supp}(A)$ to the boundary and $d_{2}$ the distance from $\operatorname{supp}(A)$ to the set of plaquettes in $E$.

The proof of Lemma 2.3 simply uses the fact that the activity of large exterior defect networks decays exponentially with their size and is done in Sect. 3. Lemma 2.4 should be plausible for the reader familiar with the method of cluster expansions and is proved in Sect. 4. We now give the

Proof of Lemma 2.2, assuming Lemmas 2.3 and 2.4. Without loss we assume that $\Lambda$ is so large that $|\Lambda| \leqq 2\left|\Lambda^{\prime}\right|\left[\Lambda^{\prime}\right.$ as in Eq. (2.5)]. By Lemma 2.3 and Eq. (1.13)

$$
\left|\langle\bar{A}\rangle_{\underline{b}}^{\Lambda}-\sum_{E:|E| \leqq|\partial \Lambda|^{1+\varrho}} p_{b}(E)\langle\bar{A}\rangle_{E}^{\Lambda}\right| \leqq\|A\| \varepsilon(|\partial \Lambda|, \varrho),
$$

where $\|\cdot\|$ denotes the supremum norm. Next we choose

$$
d_{1}=d_{2}=|\partial \Lambda|^{\rho / d}
$$

and replace $\langle\bar{A}\rangle_{E}^{\Lambda}$ in $(2.8)$ by

$$
\sum_{x \in \Lambda^{\prime \prime}} \frac{1}{\left|\Lambda^{\prime \prime}\right|}\left\langle t_{x}(A)\right\rangle_{E}^{\Lambda},
$$

where $\Lambda^{\prime \prime}$ is the set of points in $\Lambda^{\prime}$ such that the distance from the support of $t_{x}(A)$ to $\partial \Lambda$ respectively $E$ is larger than $d_{1}$ respectively $d_{2}$. This induces an error which can be bounded by

$$
2\|A\| \frac{d_{1}^{d}|\partial \Lambda|+d_{2}^{d}|E|}{\left|\Lambda^{\prime}\right|} \leqq 8\|A\| \frac{|\partial \Lambda|^{1+2 \varrho}}{|\Lambda|} .
$$

But for $x \in \Lambda^{\prime \prime}$ we can apply Lemma 2.4. Then we apply Lemma 2.3 again and use the fact that $\sum_{E}^{\prime} p_{b}(E)=1$ to get

$$
\left|\langle\bar{A}\rangle_{\underline{b}}^{\Lambda}-\langle A\rangle_{\mathbb{1}}\right| \leqq \tilde{\varepsilon}\left(A,|\partial \Lambda|^{\varrho / d}\right)+2\|A\| \varepsilon(|\partial \Lambda|, \varrho)+8\|A\| \frac{|\partial \Lambda|^{1+2 \varrho}}{|\Lambda|} .
$$

Choosing $\varrho$ small enough this implies Lemma 2.2.

\section{Analysis of the Probabilities $p_{b}(E)$}

In this section we analyze the probabilities

$$
p_{b}(E)=z(E) Z_{E} / \tilde{Z}_{b}^{\Lambda} .
$$

We will first prove Lemma 2.3 which was used in Sect. 2 and then derive some results needed for Sects. 5 and 6. 
Recall the definition of $Z_{E}$,

$$
Z_{E}=\sum_{D}^{\prime \prime} z(D)
$$

where $\sum$ "goes over all interior defect networks satisfying the Bianchi-identity and being compatible with $E$. If one drops the last restriction one gets an upper bound for $Z_{E}$. With the definitions of Sect. 1 we see that we can resum to obtain

$$
Z_{E} \leqq \tilde{Z}_{\mathbb{1}}^{\Lambda},
$$

where $\tilde{Z}_{\mathbb{1}}^{A}$ is the partition function with b.c. $b=\mathbb{1}$.

Next we bound the ratio $\tilde{Z}_{\mathbb{1}}^{A} / \tilde{Z}_{b}^{A}$. To each gauge field configuration $g$ contributing to $\tilde{Z}_{1}^{\Lambda}$ corresponds a gauge field configuration $g^{\prime}$ contributing to $Z_{b}^{\tilde{\Lambda}}$. we simply have to change the gauge field for links in $\partial \Lambda$. It is not hard to see that this correspondence is $1-1$ and that the plaquette variables for $g$ and $g^{\prime}$ differ on not more than $d(d-1)|\partial \Lambda|$ plaquettes. This implies

$$
\tilde{Z}_{\mathbb{1}}^{\Lambda} \leqq K^{|\partial \Lambda|} \tilde{Z}_{b}^{\Lambda}
$$

with

$$
K=\max _{g \in G} e^{d(d-1) s(g)},
$$

and inserted into (3.1) and (3.2) yields

$$
p_{b}(E) \leqq K^{|\partial \Lambda|} z(E) \text {. }
$$

To complete the proof of Lemma 2.3 we have to bound

$$
Q_{b}(M)=\sum_{E:|E| \geqq M}^{\prime} z(E)
$$

with $M=|\partial \Lambda|^{1+\varrho}$. We first bound the sum over defect networks $E$ with fixed support $T$ by $\exp (-b|T|)$, where $|T|$ denotes the number of plaquettes in $T$. Inserting this in (3.5) yields

$$
Q_{b}(M) \leqq \sum_{T:|T| \geqq M}^{\prime} e^{-b|T|},
$$

where $\Sigma^{\prime}$ goes over possible supports $T$ of exterior defect networks. Let now $P_{1}, \ldots, P_{n}$ be the (pos. oriented) plaquettes in $\partial \Lambda$. Due to Definition 1.4 each such $T$ can be written as a union of coconnected set $T_{1}, \ldots, T_{n}$, some of them possibly empty, such that $T_{i}$ contains $P_{i}$ if it isn't empty. If we fix the sizes of $T_{1}, \ldots, T_{n}$ to be $s_{1}, \ldots, s_{n}$ there are at most (see Lemma 1.5) $K_{0}^{s_{1}+s_{2}+\ldots+s_{n}}$ such sets $T_{1}, \ldots, T_{n}$, and we can bound

$$
\begin{aligned}
Q_{b}(M) & \leqq \sum_{s=M}^{\infty} e^{-s b} \sum_{\substack{1, \ldots, s_{n} \geq 0 \\
\Sigma s_{i}=s}} K_{0}^{s} \\
& =\sum_{s=M}^{\infty}\left(K_{0} e^{-b}\right)^{s}\left(\begin{array}{c}
n+s-1 \\
n-1
\end{array}\right) \\
& \leqq \sum_{s=M}^{\infty}\left(K_{0} e^{-b}\right)^{s} \frac{(n+s-1)^{n-1}}{(n-1) !} .
\end{aligned}
$$

To continue we need the simple 
Lemma 3.1 [6]. Let $0<x<1$. Then

$$
\sum_{s=N}^{\infty} s^{p} x^{s} \leqq p ! x^{N} \frac{1+p N^{p}}{(1-x)^{p+1}}
$$

for all integers $N, p \geqq 0$.

The proof is done by induction and can be found in [6].

This can be used to estimate (3.6) further: we do the change of variables $s+n-1$ $\rightarrow s$ and apply Lemma 3.1 to (3.6) with $N=M+n-1$ to get

$$
Q_{b}(M) \leqq\left(K_{0} e^{-b}\right)^{M} \frac{1+(n-1)(M+n-1)^{n-1}}{\left(1-K_{0} e^{-b}\right)^{n}} \leqq\left(K_{0} e^{-b}\right)^{M}\left(\frac{M+n-1}{1-K_{0} e^{-b}}\right)^{n} .
$$

Put $M=|\partial \Lambda|^{1+\varrho}$. Then (3.4), (3.5), and (3.7) together with the estimate $n \leqq(d-1)$ $(d-2)|\partial \Lambda|$ yield Lemma 2.3 .

Whereas the estimate (3.2) was good enough for the purpose of Lemma 2.3, a more careful analysis of the ratio

$$
e^{-V_{\Lambda}(E)} \equiv Z_{E} / \tilde{Z}_{\mathbb{1}}^{\Lambda}
$$

is needed for the construction of a nontranslation invariant state in Sect. 5 respectively 6 . More precisely we need a decomposition

$$
V_{\Lambda}(E)=\sum_{p \in E} v(P, E, \Lambda)
$$

and bounds on $v(P, E, \Lambda)$ which are uniform in $E$ and $\Lambda$, see Lemma 3.3 and 3.4 below.

For this purpose we derive a cluster expansion for $V_{A}(E)$. We first need some definitions. Let $\Lambda_{P}^{\infty}$ be the set of positive orientated plaquettes in $\mathbb{Z}^{d}$.

Definition 3.2. A polymer $\gamma$ is a function with finite support from $\Lambda_{P}^{\infty}$ into $G$, such that

i) the Bianchi-identity is fulfilled for all cubes in $\mathbb{Z}^{d}$,

ii) the support of $\gamma$ is a coconnected set of plaquettes, not empty. We say $P$ is a plaquette in $\gamma$ if $\gamma(P) \neq \mathbb{1}$, the number of plaquettes $P \in \gamma$ is called the size $|\gamma|$ of $\gamma$ and $\gamma$ is called a polymer in $\Lambda$, if the support of $\gamma$ is contained in $\Lambda_{P}$ and $\gamma(P)=\mathbb{1}$ for all plaquettes in $\partial \Lambda$.

A polymer $\gamma$ and a plaquette $P$ are called compatible if none of the plaquettes in $\gamma$ is coconnected to $P$. Two polymers $\gamma, \gamma^{\prime}$ are called compatible if all the plaquettes in $\gamma$ are compatible with $\gamma^{\prime}$. A polymer $\gamma$ and an exterior defect network $E$ are compatible if any plaquette in the support of $E$ is compatible with $\gamma$.

The activity $z(\gamma)$ of $\gamma$ is defined by

$$
z(\gamma)=\prod_{P \in \Lambda_{P}^{\infty}} e^{-s(\gamma(P))}
$$

the activity $z_{\Lambda}(\gamma)$ is the same as $z(\gamma)$ if $\gamma$ is a polymer in $\Lambda$, and zero otherwise. The activity $z_{E}(\gamma)$ is the same as $z_{\Lambda}(\gamma)$ if $\gamma$ is compatible with $E$ and zero otherwise. 
It is evident that any defect network contributing to $Z_{E}$ respectively $\tilde{Z}_{\mathbb{1}}^{\Lambda}$ decomposes into a set of pairwise compatible polymers in $\Lambda$. It follows that

$$
\begin{aligned}
& Z_{E}=1+\sum_{n=1}^{\infty} \sum_{\left\{\gamma_{1}, \ldots, \gamma_{n}\right\}_{c}} \prod_{i=1}^{n} z_{E}\left(\gamma_{i}\right), \\
& \tilde{Z}_{1}^{\Lambda}=1+\sum_{n=1}^{\infty} \sum_{\left\{\gamma_{1}, \ldots, \gamma_{n_{3}}\right\}_{c}} \prod_{i=1}^{n} z_{\Lambda}\left(\gamma_{i}\right),
\end{aligned}
$$

where $\left\{\gamma_{1}, \ldots, \gamma_{n}\right\}_{c}$ denotes a set of pairwise compatible polymers. We can now apply the method of cluster expansions (see [4]) and obtain

$$
\begin{aligned}
& \ln Z_{E}=\sum_{n=1}^{\infty} \sum_{\gamma_{1}, \ldots, \gamma_{n}} \frac{a\left(\left\{\gamma_{i}\right\}\right)}{n !} \prod_{i=1}^{n} z_{E}\left(\gamma_{i}\right), \\
& \ln \tilde{Z}_{\mathbb{1}}^{A}=\sum_{n=1}^{\infty} \sum_{\gamma_{1}, \ldots, \gamma_{n}} \frac{a\left(\left\{\gamma_{i}\right\}\right)}{n !} \prod_{i=1}^{n} z_{\Lambda}\left(\gamma_{i}\right),
\end{aligned}
$$

where the combinatoric coefficient $a\left(\left\{\gamma_{i}\right\}\right)$ is obtained as follows: let $G\left(\left\{\gamma_{i}\right\}\right)$ be the graph with vertices $\gamma_{1}, \ldots, \gamma_{n}$ and a line between $\gamma$ and $\gamma^{\prime}$ whenever $\gamma$ and $\gamma^{\prime}$ are incompatible. Then

$$
a\left(\left\{\gamma_{i}\right\}\right)=\sum_{c \subset G\left(\left\{\gamma_{i}\right\}\right)}(-1)^{\ell(c)}
$$

when the sum goes over connected subgraphs having the same vertex set as $G, \ell(C)$ is the number of lines in $C$. Here we only need that $a\left(\left\{\gamma_{i}\right\}\right) \neq 0$ only if the graph $G\left(\left\{\gamma_{i}\right\}\right)$ is connected.

Absolute convergence of the cluster expansions (3.11) and (3.12) for $b>\log K_{0}+\log 2.93$ is proven in the appendix. Equations (3.11) and (3.12) yield

$$
V_{\Lambda}(E)=\sum_{n=1}^{\infty} \sum_{\gamma_{1}, \ldots, \gamma_{n}} \frac{a\left(\left\{\gamma_{i}\right\}\right)}{n !}\left\{\prod_{i=1}^{n} z_{\Lambda}\left(\gamma_{i}\right)-\prod_{i=1}^{n} z_{E}\left(\gamma_{i}\right)\right\} .
$$

Since $z_{\Lambda}(\gamma)=z_{E}(\gamma)$ if $\gamma$ is compatible with $E$, we may restrict the sum to sequences $\gamma_{1}, \ldots, \gamma_{n}$ where at least one $\gamma$ is incompatible with $E$. Let $\tilde{a}\left(\left\{\gamma_{i}\right\}\right)=a\left(\left\{\gamma_{i}\right\}\right)$ if at least one $\gamma_{i}$ is incompatible with $E$ and $\tilde{a}\left(\left\{\gamma_{i}\right\}\right)=0$ if not. Then

$$
V_{\Lambda}(E)=\sum_{n=1}^{\infty} \sum_{\gamma_{1}, \ldots, \gamma_{n}} \frac{\tilde{a}\left(\left\{\gamma_{i}\right\}\right)}{n !} \prod_{i=1}^{n} z_{\Lambda}\left(\gamma_{i}\right),
$$

because $z_{E}(\gamma)=0$ if $\gamma$ is incompatible with $E$.

Let $N_{E}\left(\gamma_{1}, \ldots, \gamma_{n}\right)$ be the number of polymers in $\left\{\gamma_{1}, \ldots, \gamma_{n}\right\}$ which are incompatible with $E$. Then

$$
N_{E}\left(\gamma_{1}, \ldots, \gamma_{n}\right) \tilde{a}\left(\gamma_{1}, \ldots, \gamma_{n}\right)=\sum_{k=1}^{n} a_{k}\left(\gamma_{1}, \ldots, \gamma_{n}\right),
$$

where $a_{k}\left(\gamma_{1}, \ldots, \gamma_{n}\right)=a\left(\gamma_{1}, \ldots, \gamma_{n}\right)$ if $\gamma_{k}$ is incompatible with $E$ and $a_{k}\left(\gamma_{1}, \ldots, \gamma_{n}\right)=0$ if not. Inserting this in (3.13) we get

$$
\begin{aligned}
V_{\Lambda}(E) & =\sum_{n=1}^{\infty} \sum_{\gamma_{1}, \ldots, \gamma_{n}} \sum_{k=1}^{n} \frac{1}{n !} \frac{a_{k}\left(\gamma_{1}, \ldots, \gamma_{n}\right)}{N_{E}\left(\gamma_{1}, \ldots, \gamma_{n}\right)} \prod_{i=1}^{n} z_{\Lambda}\left(\gamma_{i}\right) \\
& =\sum_{\gamma_{1}}^{\prime} \sum_{n=1}^{\infty} \sum_{\gamma_{2}, \ldots, \gamma_{n}} \frac{1}{(n-1) !} \frac{a\left(\left\{\gamma_{i}\right\}\right)}{N_{E}\left(\gamma_{1}, \ldots, \gamma_{n}\right)} \prod_{i=1}^{n} z_{\Lambda}\left(\gamma_{i}\right),
\end{aligned}
$$

where $\Sigma^{\prime}$ goes over all polymers $\gamma_{1}$ which are incompatible with $E$. 
Let $M_{E}\left(\gamma_{1}\right)$ be the number of plaquettes $P$ in the support of $E$, which are incompatible with $\gamma_{1}$. By an argument similar to the one leading to (3.14) one gets

$$
V_{\Lambda}(E)=\sum_{P \in E} \sum_{n=1}^{\infty} \frac{1}{(n-1) !} \sum_{\gamma_{1}, \ldots, \gamma_{n}}^{*} \frac{a\left(\left\{\gamma_{i}\right\}\right)}{M_{E}\left(\gamma_{1}\right) N_{E}\left(\gamma_{1}, \ldots, \gamma_{n}\right)} \prod_{i=1}^{n} z_{\Lambda}\left(\gamma_{i}\right),
$$

where the sum $\Sigma^{*}$ goes over polymers $\gamma_{1}, \ldots, \gamma_{n}$ such that $\gamma_{1}$ is incompatible with $P$. Equation (3.15) is the desired decomposition of $V_{A}(E)$.

Lemma 3.3. For $b>\log K_{0}+\log 2.93$ the probabilities $p_{b}(E)$ can be written in the form

$$
p_{\underline{b}}(E)=\bar{Z}_{\underline{b}}^{-1} z(E) e^{-V_{\Lambda}(E)}
$$

with the obvious normalization factor $\bar{Z}_{b}$ and

$$
\begin{gathered}
V_{\Lambda}(E)=\sum_{P \in E} v(P, E, \Lambda), \\
v(P, E, \Lambda)=\sum_{n=1}^{\infty} \frac{1}{(n-1) !} \sum_{\gamma_{1}, \ldots, \gamma_{n}}^{*} \frac{a\left(\left\{\gamma_{i}\right\}\right)}{M_{E}\left(\gamma_{1}\right) N_{E}\left(\gamma_{1}, \ldots, \gamma_{n}\right)} \prod_{i=1}^{n} z_{\Lambda}\left(\gamma_{i}\right) .
\end{gathered}
$$

Proof. The lemma follows directly from (3.1), (3.8), and (3.15).

Remark. The above decomposition of $p_{b}(E)$ is a decomposition into the factor

$$
z(E)=\exp \left\{-\sum_{P \in E} s(E(P))\right\}
$$

where $s$ is a "one body potential" and the factor $\exp \left\{-V_{A}(E)\right\}$, which describes the interaction of different plaquettes in $E$. This interaction is mediated via the polymers $\gamma_{1}, \ldots, \gamma_{n}$, i.e. via the interior defect network.

The fact that interaction $V_{A}$ plays only a minor role is important for the purpose of Sect. 6 and is formulated in the following lemma. We need some notations. Suppose the plaquette $\widetilde{P}$ is a translate of $P, \widetilde{P}=t_{x}(P)$. Let $y$ be the center of $\widetilde{P}$ and $K(y, r)=\left\{z \in \mathbb{Z}^{d}|| z-y \mid \leqq r\right\}$. Following [3] we define $d(P, E, \Lambda ; \widetilde{P}, \widetilde{E}, \widetilde{\Lambda})$ to be the maximal $r$, for which

i) $(\Lambda+x) \cap K(y, r)=\tilde{\Lambda} \cap K(y, r)$, and

ii) $E\left(P^{\prime}\right)=\widetilde{E}\left(t_{x}\left(P^{\prime}\right)\right)$ as long as the distance between the centers of $P$ and $P^{\prime}$ is not larger than $r$.

Remark. Heuristically $d(\ldots)$ is the maximal radius $r$, for which an observer sitting at $P$ and observing $\Lambda$ and $E$ up to distance $r$, and an observer sitting at $\widetilde{P}$ and observing $\tilde{\Lambda}$ and $\widetilde{E}$ up to distance $r$, see the same things.

Lemma 3.4. Suppose $b>\log K_{0}+\log 2.93$ and let

$$
g(b)=\left.\frac{\varepsilon^{4}}{1-\varepsilon}\left(1-\frac{32 \varepsilon^{4}}{(1-\varepsilon)^{2}}\right)^{-1}\right|_{\varepsilon=K_{0} e^{-b}} .
$$

Then

$$
|v(P, E, \Lambda)| \leqq g(b),
$$


and for $m<b-\log K_{0}-\log 2.93$,

$$
|v(P, E, \Lambda)-v(\tilde{P}, \tilde{E}, \tilde{\Lambda})| \leqq 2 g(b-m) e^{-m d(P, E, \Lambda ; \tilde{P}, \tilde{E}, \tilde{\Lambda})},
$$

if $\tilde{P}$ is a translate of $P$.

Proof. From Lemma 3.3 it follows that

$$
|v(P, E, \Lambda)| \leqq \sum_{n=1}^{\infty} \frac{1}{(n-1) !} \sum_{\gamma_{1}, \ldots, \gamma_{n}}^{*} a\left(\left\{\gamma_{i}\right\}\right) \prod_{i=1}^{n}\left|z\left(\gamma_{i}\right)\right|,
$$

which is bounded by $g(b)$ in the same way as Lemma A.3 is proven in the appendix. To prove the second inequality of the lemma we may assume without loss that $P=\tilde{P}$, because $v(P, E, \Lambda)$ as well as $d(P, E, \Lambda ; \widetilde{P}, \widetilde{E}, \tilde{\Lambda})$ do not change if we translate $P, E$, and $\Lambda$ by the same vector $x \in \mathbb{Z}^{d}$. Using again Lemma 3.3 we get

$$
\begin{gathered}
|v(P, E, \Lambda)-v(\tilde{P}, \tilde{E}, \tilde{\Lambda})| \leqq \sum_{n=1}^{\infty} \frac{1}{(n-1) !} \sum_{\gamma_{1}, \ldots, \gamma_{n}}^{*}\left|a\left(\left\{\gamma_{i}\right\}\right)\right| \\
\quad \cdot\left|\frac{z_{\Lambda}\left(\gamma_{1}\right) \ldots z_{\Lambda}\left(\gamma_{n}\right)}{M_{E}\left(\gamma_{1}\right) N_{E}\left(\gamma_{1}, \ldots, \gamma_{n}\right)}-\frac{z_{\tilde{\Lambda}}\left(\gamma_{1}\right) \ldots z_{\tilde{\Lambda}}\left(\gamma_{n}\right)}{M_{\tilde{E}}\left(\gamma_{1}\right) N_{\tilde{E}}\left(\gamma_{1}, \ldots, \gamma_{n}\right)}\right| .
\end{gathered}
$$

Now it is evident that the last factor on the right-hand side can only be different from 0 , if either

i) there is a polymer $\gamma$ in $\left\{\gamma_{1}, \ldots, \gamma_{n}\right\}$ which lies in $\Lambda$ and not in $\tilde{\Lambda}$ or vice versa, or

ii) there is a polymer $\gamma$ in $\left\{\gamma_{1}, \ldots, \gamma_{n}\right\}$ which is incompatible with $E$ and not with $\tilde{E}$ or vice versa.

Using the fact that $a\left(\left\{\gamma_{i}\right\}\right)$ is only different from 0 if the plaquettes corresponding to $\gamma_{1}, \ldots, \gamma_{n}$ form a coconnected set, and that $\gamma_{1}$ coconnects to $P=\tilde{P}$, it follows that only sequences $\gamma_{1}, \ldots, \gamma_{n}$ contribute to the right-hand side of (3.16), for which

$$
\sum_{i=1}^{n}\left|\gamma_{i}\right| \geqq d(P, E, \Lambda ; \tilde{P}, \tilde{E}, \tilde{\Lambda})
$$

We therefore may extract a factor

$$
e^{-m d(P, E, \Lambda ; \tilde{P}, \tilde{E}, \tilde{\Lambda})}
$$

from (3.16) and bound the resulting sum by $2 g(b-m)$, which proves the second inequality of the lemma.

Remark. Dobrushin proved a similar lemma in [3], but he used an expansion directly for $\ln Z_{E}$ instead of for $V(E)$. This makes his definition of $v(\ldots)$ [there called $\left.f_{\beta}(\ldots)\right]$ and proof of the corresponding lemma more involved.

\section{Analysis of the Expectation Values $\langle A\rangle_{E}^{A}$}

In this section we analyze the conditional expectation values $\langle A\rangle_{E}^{A}$ with fixed exterior defect network $E$. We first will prove Lemma 2.4 which was used in Sect. 2 and then state a lemma which will be needed in Sect. 6 . For the proof of these 
lemmas we use a cluster expansion for $\langle A\rangle_{E}^{A}$ which will be derived in the first part of this section.

It is convenient to study modified partition functions

$$
Z_{E}^{A}(1+A)=\sum_{D}^{\prime \prime} z(D)(1+A(E, D))
$$

where the sum goes over interior defect networks, satisfying the Bianchi-identity and compatible with $E .\langle A\rangle_{E}^{A}$ is obtained by differentiation:

$$
\langle A\rangle_{E}^{A}=\left.\frac{d}{d \alpha}\right|_{\alpha=0} \ln Z_{E}^{A}(1+\alpha A) .
$$

It is clear that we have to change our definition of a polymer $\gamma$, because it might be coconnected through the support of $A$. More precisely: assume without loss (by gauge invariance) that the support of $A$ consists of possibly splitting loops. Choose surfaces of plaquettes spanned into these loops and denote the resulting set of plaquettes $\mathscr{A}$. Clearly $A(E, D)$ depends only on the value of $E$ and $D$ on plaquettes in $\mathscr{A}$.

Definition 4.1. A polymer $\gamma$ corresponding to $A$ is a function with finite support from $\Lambda_{P}^{\infty}$ into $G$, such that the Bianchi-identity is fulfilled for all cubes in $\mathbb{Z}^{d}$, and the support of $\gamma$ decomposes into coconnectivity components, each of them containing a plaquette in $\mathscr{A}$. A polymer $\gamma$ is either a polymer corresponding to $A$ (write $n_{A}(\gamma)=1$ ) or a polymer according to Definition 3.2 (write $n_{A}(\gamma)=0$ and call it ordinary polymer in the sequel).

Two polymers $\gamma, \gamma^{\prime}$ are called incompatible if

i) $n_{A}(\gamma)=n_{A}\left(\gamma^{\prime}\right)=1$ or

ii) $n_{A}(\gamma)=n_{A}\left(\gamma^{\prime}\right)=0$ and $\gamma, \gamma^{\prime}$ are incompatible according to Definition 3.2 or

iii) $n_{A}(\gamma)=1, n_{A}\left(\gamma^{\prime}\right)=0$ and $\gamma^{\prime}$ is incompatible with a plaquette in the support of $\gamma$ or contains a plaquette in $\mathscr{A}$.

$\gamma$ is compatible with the exterior defect network $E$, if the support of $\gamma$ contains no plaquette which coconnects to $E$. The activity $z_{A, E}(\gamma)$ is defined by

$$
z_{A, E}(\gamma)=A(E, \gamma)^{n(\gamma)} \prod_{P \in \Lambda_{P}^{\infty}} e^{-s(\gamma(P))}
$$

if $\gamma$ is compatible with $E$ and lies in $\Lambda$, and is set to zero otherwise.

It is evident that each interior defect network contributing to (4.1) decomposes into a set $\left\{\gamma_{1}, \ldots, \gamma_{m}\right\}_{c}$ of pairwise compatible polymers and that (4.1) becomes

$$
Z_{E}^{\Lambda}(1+A)=\sum_{m=0}^{\infty} \sum_{\left\{\gamma_{1}, \ldots, \gamma_{m}\right\}_{c}} \prod_{i=1}^{m} z_{A, E}\left(\gamma_{i}\right)
$$

where an empty product has to be interpreted as one. Equation (4.4) is of the same form as (3.9) and we can again apply the method of cluster expansions for polymer systems to obtain

$$
\ln Z_{E}^{A}(1+A)=\sum_{m=1}^{\infty} \sum_{\gamma_{1}, \ldots, \gamma_{m}} \frac{a\left(\left\{\gamma_{i}\right\}\right)}{m !} \prod_{i=1}^{m} z_{A, E}\left(\gamma_{i}\right),
$$

and using (4.2)

$$
\langle A\rangle_{E}^{\Lambda}=\sum_{m=1}^{\infty} \sum_{\gamma_{1}, \ldots, \gamma_{m}}^{\prime} \frac{a\left(\left\{\gamma_{i}\right\}\right)}{m !} \prod_{i=1}^{m} z_{A, E}\left(\gamma_{i}\right)
$$


where the sum $\Sigma^{\prime}$ goes over terms linear in $A$, i.e. over $\gamma_{1}, \ldots, \gamma_{m}$ such that $n_{A}\left(\gamma_{i}\right)=1$ for exactly one $\gamma_{i}$. In the same way

$$
\langle A\rangle_{\mathbb{1}}^{\Lambda}=\sum_{m=1}^{\infty} \sum_{\gamma_{1}, \ldots, \gamma_{m}}^{\prime} \frac{a\left(\left\{\gamma_{i}\right\}\right)}{m !} \prod_{i=1}^{m} z_{A, \mathbb{1}}\left(\gamma_{i}\right),
$$

where for polymers $\gamma$ in $\Lambda$

$$
z_{A, \mathbb{1}}(\gamma) \equiv A(\mathbb{1}, \gamma)^{n(\gamma)} \prod_{P \in \Lambda_{P}^{\infty}} e^{-s(\gamma(P))}
$$

and for polymers $\gamma$ not in $\Lambda z_{A, \mathbb{1}}(\gamma)=0$. Absolute convergence uniform in $\Lambda$ and $E$ is shown for the cluster expansions (4.5) and (4.6) in the appendix, provided $b>\log K_{0}+\log 2.93$.

Remark. This shows that the thermodynamic limit in (4.6) can be taken term by term, yielding

$$
\langle A\rangle_{\mathbb{1}}=\sum_{m=1}^{\infty} \sum_{\gamma_{1}, \ldots, \gamma_{m}}^{\prime} \frac{a\left(\left\{\gamma_{i}\right\}\right)}{m !} \prod_{i=1}^{m} z_{A}\left(\gamma_{i}\right)
$$

where

$$
z_{A}(\gamma) \equiv A(\mathbb{1}, \gamma)^{n(\gamma)} \prod_{P \in \Lambda_{P}^{\infty}} e^{-s(\gamma(P))}
$$

for all polymers $\gamma$.

Lemma 2.4 is now a corollary of (4.5) and (4.8):

Proof of Lemma 2.4. Let $r_{1}$ respectively $r_{2}$ be the distance from $\mathscr{A}$ to the boundary respectively to the support of $E$. Since $\min \left(r_{1}, r_{2}\right) \rightarrow \infty$ if $d_{1}$ and $d_{2}$ go to $\infty$, it is enough to prove Lemma 2.4 with $d_{i}$ replaced by $r_{i}$. From (4.5) and (4.8) it follows that

$$
\left|\langle A\rangle_{E}^{A}-\langle A\rangle_{\mathbb{1}}\right| \leqq \sum_{m=1}^{\infty} \sum_{\gamma_{1}, \ldots, \gamma_{m}}^{\prime} \frac{a\left(\left\{\gamma_{i}\right\}\right)}{m !}\left|\prod_{i=1}^{m} z_{A, E}\left(\gamma_{i}\right)-\prod_{i=1}^{m} z_{A}\left(\gamma_{i}\right)\right| .
$$

The difference on the right-hand side is only different from 0 , if one of the $\gamma_{i}$ 's is incompatible with $E$ or doesn't lie in $\Lambda$. On the other hand there is a $\gamma_{i}$ in the sequence $\gamma_{1}, \ldots, \gamma_{n}$ with $n_{A}\left(\gamma_{i}\right)=1$, and the plaquettes corresponding to the supports of $\gamma_{1}, \ldots, \gamma_{n}$ form a coconnected (possibly through $\mathscr{A}$ ) set of plaquettes. Therefore for each term contributing to (4.10),

$$
\sum_{i=1}^{m}\left|\gamma_{i}\right| \geqq \min \left(r_{1}, r_{2}\right) \text {. }
$$

Now choose $M<b-\log K_{0}-\log 2.93$, extract a factor exp $\left(-M \min \left(r_{1}, r_{2}\right)\right)$ from the right-hand side of (4.10) and apply Lemma A.5 to the remaining sum. This yields

$$
\left|\langle A\rangle_{E}^{\Lambda}-\langle A\rangle_{\mathbb{1}}\right| \leqq \text { const } e^{-M \min \left(r_{1}, r_{2}\right)},
$$

where the constant depends in a translation invariant way on $A$. This implies Lemma 2.4.

We finally prove a lemma which will be needed in Sect. 6 . 
Lemma 4.2. Let $\chi_{q}(\cdot)$ be the character of an irreducible representation of $G$. Then

$$
\left\langle\chi_{q}\left(g_{\partial P}\right)\right\rangle_{E}^{A}=\chi_{q}(E(P))
$$

if $P$ lies in the support of $E$, and

$$
\left|\left\langle\chi_{q}\left(g_{\partial P}\right)\right\rangle_{E}^{A}-\chi_{q}(\mathbb{1})\right| \leqq K_{1} e^{-b}
$$

if not. $K_{1}$ is a numerical constant proportional to $K_{0}$.

Remark. This is the counterpart to Lemma 3.4 for expectation values. It shows that the fluctuations due to the existence of interior defect networks are small and that the expectation values $\left\langle\chi_{q}\left(g_{\partial P}\right)\right\rangle_{E}^{\Lambda}$ are roughly already given by the value of $E$ at $P$.

Proof. Equation (4.11) is evident. If we choose $K_{1} \geqq 6 K_{0},(4.12)$ is trivial for $b \leqq \log K_{0}+\log 3$. So we may assume without $\operatorname{loss} b>\log K_{0}+\log 3$. Note that $\chi_{q}(\mathbb{1})$ is a term in the series $(4.5)$ for $\left\langle\chi_{q}\left(g_{\partial P}\right)\right\rangle_{E}^{A}$ if $E(P)=\mathbb{1}$ :

$$
\chi_{q}(\mathbb{1})=z_{A, E}\left(\gamma_{0}\right)=a\left(\left\{\gamma_{0}\right\}\right) z_{A, E}\left(\gamma_{0}\right)
$$

where $\gamma_{0}$ is the polymer with $n_{A}\left(\gamma_{0}\right)=1$ and empty support. The other terms in (4.5) and therefore all terms in the series for the left-hand side of (4.12) are terms for which $\sum\left|\gamma_{i}\right| \geqq 1$. We extract a factor $3 K_{0} e^{-b}$ and bound the resulting sum using Lemma A.5. This yields $\left(r_{0} \geqq 4\right)$

$$
\left|\left\langle\chi_{q}\left(g_{\partial P}\right)\right\rangle_{E}^{A}-\chi_{q}(\mathbb{1})\right| \leqq 3 K_{0} e^{-b} \frac{e(2 d) !}{\left(1-e \varepsilon_{0}\right)^{2 d}}\left(1-\frac{32 \varepsilon_{0}^{4}}{\left(1-\varepsilon_{0}\right)^{2}}\right)^{-1}
$$

with $\varepsilon_{0}=\left(K_{0} e^{-b}\right) e^{b} / 3 K_{0}=1 / 3$. This proves (4.12).

\section{Construction of a Non-Translation Invariant Equilibrium State for $d=4$}

As in Sect. 3, $\Lambda_{P}^{\infty}$ denotes the positively oriented plaquettes in $\mathbb{Z}^{d}$, but we restrict ourselves to $d=4$. The position $x=\left(x_{1}, x_{2}, x_{3}, x_{4}\right)$ of a plaquette $P$ is the position of its center. Its height $h$ is defined with respect to the plane $y_{1}=y_{2}=-1 / 2$ : $h=\left(h_{1}, h_{2}\right)=\left(x_{1}+1 / 2, x_{2}+1 / 2\right)$. Note that a plaquette $P$ is parallel to the $k \ell$-plane, if the $k$ - and $\ell$-coordinates $x_{k}$ and $x_{\ell}$ of its position $x$ are half-integer valued. In particular a plaquette with height zero is parallel to the 12-plane.

The goal of this and the following section is to prove

Theorem 5.1. In $d=4$ there is a constant $J_{0}>0$ and a function $f(J)$ going to zero with $J \rightarrow \infty$, both only depending on the gauge group $G$ considered, such that for any $J>J_{0}$ and any $g_{0} \in G$ with $s\left(g_{0}\right)=\min _{g \neq \mathbb{1}} s(g)$ there is an infinite volume equilibrium state $\langle\cdot\rangle$, such that

$$
\left|\left\langle\chi_{q}\left(g_{\partial P}\right)\right\rangle-\chi_{q}\left(g_{0}\right)\right| \leqq f(J)
$$

if $P$ is a plaquette with height 0 and

$$
\left\langle\chi_{q}\left(g_{\partial P}\right)\right\rangle-\chi_{q}(\mathbb{1}) \mid \leqq f(J)
$$

if not. $\chi_{q}(\cdot)$ denotes the character of an arbitrary irreducible representation of $G$. 
Remarks. i) Clearly the state $\langle\cdot\rangle$ breaks translation invariance in the 1- and 2 -direction for large $J$. Without loss we can assume translation invariance in the 3and 4-direction (if not we get it by averaging).

ii) If we interpret the 4-direction as (imaginary) time, the state $\langle\cdot\rangle$ describes a localized magnetic flux tube parallel to the 3 -axis, starting and ending at infinity.

iii) The theorem remains true for infinite discrete abelian groups, as long as $2 b-b_{0}$ goes to infinity with $J$, in addition to condition (1.4) of Sect. 1. (See the remark at the end of Sect. 6.) This applies for example to the $\mathbb{Z}$-gauge theory with interaction $s(z)=J z^{2}$.

The organization of Sects. 5 and 6 is as follows: we first introduce the b.c. forcing an exterior defect network into the system, such that the minimal exterior defect network corresponding to these b.c. has its support on the plaquettes in $\Lambda_{P}$ with height zero. The thermodynamic limit of the corresponding finite volume states $\langle\cdot\rangle_{A}$ will be the state $\langle\cdot\rangle$ of Theorem 5.1 . To show that this state really breaks translation invariance we have to show the bounds of Theorem 5.1 for the states $\langle\cdot\rangle_{A}$ uniformly in the volume $\Lambda$ (Theorem 5.2). With the preparations done in Sects. 3 and 4 (in particular Lemma 4.2) it is enough to show that the exterior defect network does not fluctuate too much away from the minimal one (see Lemma 5.3 below for the precise statement). The proof of this lemma will be given in Sect. 6 generalizing the arguments of Dobrushin [3].

Consider the following box $\Lambda_{0} \subset \mathbb{Z}^{4}$ of linear extensions $L_{1}, L_{2}, L_{3}, L_{4}$ :

$$
\Lambda_{0}=\left\{x \in \mathbb{Z}^{d} \mid-\frac{L_{i}}{2} \leqq x_{i}<\frac{L_{i}}{2}, i=1, \ldots, 4\right\},
$$

and the enlarged lattice $\bar{\Lambda}_{0}$ obtained from $\Lambda_{0}$ by replacing $L_{3}, L_{4}$ by $L_{3}+2, L_{4}+2$. Define the position and height of a positively oriented link in $\Lambda_{0}$ respectively $\bar{\Lambda}_{0}$ in the same way as for the plaquettes in $\Lambda_{P}^{\infty}$. Note that a link is parallel to the $i$-axis if the $i$ 's component of its position is half-integer valued and that the position of a plaquette $P$ containing a link with position $x$ is obtained from $x$ by adding plus or minus $1 / 2$ to one of the integer-valued coordinates of $x$. The b.c. $b$ on $\partial \Lambda_{0}$ are defined as follows:

$$
\begin{array}{ll}
b_{x y}=g_{0} & \begin{array}{l}
\text { for positively oriented links }\langle x y\rangle \text { in } \partial \Lambda_{0} \text { with } \\
\text { height } h=(0, n+1 / 2) \text { for some integer } n \geqq 0,
\end{array} \\
b_{x y}=\mathbb{1} & \text { for all other positively oriented links }\langle x y\rangle \text { in } \partial \Lambda_{0} .
\end{array}
$$

With the above remarks it is easy to see that for any positively oriented plaquette $P$ in $\partial \Lambda_{0}$,

$$
\begin{array}{ll}
b_{\partial P}=g_{0} & \text { if the height of } P \text { is zero, } \\
b_{\partial P}=\mathbb{1} & \text { if not } .
\end{array}
$$

From (5.4) and the Bianchi-identity it follows that the minimal defect network corresponding to these b.c. is the defect network $E_{0}$ with

$$
\begin{array}{ll}
E_{0}(P)=g_{0} & \begin{array}{l}
\text { if } P \text { is a positively oriented plaquette in } \Lambda_{0} \text { with } \\
\text { height } h=0 \text { and }
\end{array} \\
E_{0}(P)=\mathbb{1} & \begin{array}{l}
\text { if } P \text { is a positively oriented plaquette in } \Lambda_{0} \text { with } \\
\text { height } h \neq 0 .
\end{array}
\end{array}
$$


Consider the finite volume expectation values $\langle\cdot\rangle_{b}^{\Lambda_{0}}$ corresponding to the b.c. (5.3). We want to do the thermodynamic limit $L_{1}, L_{2} \rightarrow \underset{b}{\rightarrow} \infty$ in the 1- and 2-direction.

Absolute convergence of the cluster expansion (4.5) for $\langle A\rangle_{E}^{A_{0}}$ uniform in $L_{1}, L_{2}$ yields the existence of the limit $L_{1}, L_{2} \rightarrow \infty$ for $\langle A\rangle_{E}^{\Lambda_{0}}$ because the limit can be done term by term in (4.5) which is easy. The same argument applies (see (3.15)) to $z(E) \exp \left\{-V_{\Lambda_{0}}(E)\right\}$. Finally we consider (see Lemma 3.3)

$$
\bar{Z}_{\Lambda_{0}}=\sum_{E}^{\prime} z(E) \exp \left\{-V_{\Lambda_{0}}(E)\right\},
$$

but the sum is again absolutely convergent uniformly in $L_{1}, L_{2}$. We get existence of the limit for expectation values and probabilities with fixed exterior defect network. Absolute and uniform convergence of (1.13) finally gives the existence of the limit

$$
\langle\cdot\rangle_{\Lambda} \equiv \lim _{L_{1}, L_{2} \rightarrow \infty}\langle\cdot\rangle_{\underset{\sim}{A}}^{A_{0}}
$$

if only (4.5) and (3.15) are absolutely and uniformly convergent, i.e. if $J$ is so large that $b>\log K_{0}+\log 2.93$.

Remarks. i) This defines $J_{0}$ in Theorem 5.1 .

ii) $\Lambda$ is the lattice $\Lambda=\left\{x \in \mathbb{Z}^{d} \mid-\frac{L_{i}}{2} \leqq x_{i}<\frac{L_{i}}{2}\right.$ for $\left.i=3,4\right\}$.

iii) From the above arguments it follows that Lemmas 3.3, 3.4, and 4.2 remain true in the limit $\Lambda_{0} \rightarrow \Lambda$.

Now we state

Theorem 5.2. In $d=4$ there is a function $f(J)$ depending only on the gauge group $G$ considered and going to zero with $J \rightarrow \infty$, such that for $J>J_{0}$ the states defined by (5.3) and (5.5) satisfy (5.1) and (5.2) with $\langle\cdot\rangle$ replaced by $\langle\cdot\rangle_{\Lambda}$.

Remark. If we assume the existence of the thermodynamic limit $\Lambda \rightarrow \mathbb{Z}^{4}$, i.e. $L_{3}, L_{4}$ $\rightarrow \infty$, Theorem 5.1 follows from Theorem 5.2 since the bounds in Theorem 5.2 are uniform in $\Lambda$. The restriction $J>J_{0}$ in Theorem 5.1 is necessary because otherwise the definition in (5.5) might make no sense. By a simple compactness argument we can always assume the existence of the limit $L_{3}, L_{4} \rightarrow \infty$, at least for convenient subsequences. This proves Theorem 5.1, assuming Theorem 5.2.

To state Lemma 5.3 needed to prove Theorem 5.2 we introduce some notations. From now on we fix $L_{3}$ and $L_{4}$ (i.e. $\Lambda$ ) and all bounds - if not otherwise stated - will be uniform in $\Lambda . \bar{\Lambda}$ is the lattice obtained from $\Lambda$ by replacing $L_{3}, L_{4}$ by $L_{3}+2, L_{4}+2$. It is convenient to extend the definition of an exterior defect network $E$ from $\Lambda_{P}$ to $\Lambda_{P}^{\infty}$ as follows:

$$
\begin{aligned}
& E(P)=g_{0} \quad \text { if } P \text { is a plaquette with height zero in } \bar{\Lambda}_{P} \backslash \Lambda_{P}, \\
& E(P)=\mathbb{1} \quad \text { for all other plaquettes in } \Lambda_{P}^{\infty} \backslash \Lambda_{P} .
\end{aligned}
$$

Consider the set $\Omega_{P}$ respectively $\Omega_{P}^{\infty}$ of plaquettes dual to those in $\bar{\Lambda}_{P}$ respectively $\Lambda_{P}^{\infty}$. Clearly an exterior defect network $E$ can as well be characterized by the function

$$
\varepsilon(P)=E(* P)
$$


from $\Omega_{P}^{\infty}$ into $G$, where $* P$ denotes the plaquette dual to $P$. The support $\mathscr{E} \subset \Omega_{P}^{\infty}$ of $\varepsilon$ will be called the exterior defect surface $\mathscr{E}$ or simply the surface $\mathscr{E}$. We define $\varepsilon$ also on negatively oriented plaquettes $P(\varepsilon(P)$ is simply the inverse of the value for the corresponding plaquette in $\left.\Omega_{P}^{\infty}\right)$ but $\mathscr{E}$ is always the support in $\Omega_{P}^{\infty}$.

The dual lattice $\Omega$ is the set of points which are corners of plaquettes in $\Omega_{P}$ and is part of $\mathbb{Z}_{1 / 2}^{4}$, defined by

$$
\mathbb{Z}_{1 / 2}^{4} \equiv \mathbb{Z}^{4}+\left(\frac{1}{2}, \frac{1}{2}, \frac{1}{2}, \frac{1}{2}\right) .
$$

The regular plane $\mathscr{R}_{0}$ is the set of points $x=\left(x_{1}, x_{2}, x_{3}, x_{4}\right)$ in $\mathbb{Z}_{1 / 2}^{4}$ such that $x_{1}=x_{2}=-1 / 2$ and the regular surface $\mathscr{R}$ is the intersection of $\Omega$ and $\mathscr{R}_{0}$. The set of positively oriented plaquettes in $\mathscr{R}$ is denoted $\mathscr{R}_{P}$. A plaquette in $\mathscr{R}_{P}$ is called horizontal if it is parallel to the plane $x_{1}=x_{2}=-1 / 2$ and $\pi$ denotes the canonical projection from $\Omega$ into $\mathscr{R}$. Finally we anticipate from Sect. 6 the following definition: a horizontal plaquette $P$ is called ceiling plaquette if it is a plaquette in $\mathscr{E}$ and there is no other plaquette in $\mathscr{E}$ projecting into $\pi(P)$.

\section{Lemma 5.3.}

i) If $P$ is a ceiling plaquette, then $\varepsilon(P)=g_{0}$.

ii) Let $P \in \Omega_{P}$ be a horizontal plaquette of height zero, i.e. $P \in \mathscr{R}_{P}$. Then the probability that $P$ is a ceiling plaquette is larger than $1-f_{0}(J)$, where $f_{0}(J)$ is a function depending only on the gauge group $G$ and goes to zero with $J \rightarrow \infty$.

Remark. The proof of Lemma 5.3 is given in Sect. 6. For the reader not willing to enter into the details I make some remarks. i) Is a simple consequence of the Bianchi-identity written down in the dual lattice and is left to the reader (respectively done in the next section). The strategy to prove ii) is as follows: one defines a wall in such a way that the walls of an exterior defect network describe the deviation from the minimal defect network. Furthermore one chooses the notion of compatibility such that $P \in \mathscr{R}_{P}$ is a ceiling plaquette whenever $P$ is compatible with all walls of the exterior defect network. We then have to bound the probability that the exterior defect network contains a wall which is incompatible with a given plaquette $P \in \mathscr{R}_{P}$. After complicated geometric construction - including a "renormalization group step" which consists in grouping together walls which are closed (see Definition 6.8) - this probability is shown to be small for reasons analogous to those giving small probability to non-empty contours in the twodimensional Ising system.

We close this section with the

Proof of Theorem 5.2, assuming Lemma 5.3. Theorem 5.2 is an obvious consequence of Lemmas 4.2 and 5.3 if one sets $f(J)=K_{1} f_{0}(J) e^{-b}$.

\section{Analysis of the Exterior Defect Surface}

In this section we prove Lemma 5.3 by generalizing arguments of Dobrushin [3]. The reader familiar with [3] will find many deviations from definitions and lemmas in [3]. Sometimes this is simply more convenient, sometimes also the straightforward generalization of notions and lemmas of Dobrushin would lead to incorrect statements. We begin with some definitions. 
Two plaquettes (links) in $\Omega$ are called connected if they share a common link (point). A link $\ell$ and a plaquette $P$ (a point $x$ ) in $\Omega$ are called connected if $\ell \in \partial P$ $(x \in \partial \ell)$. A point and a plaquette in $\Omega$ are never directly connected, but they may be connected through a link in $\Omega$. Recall that $\pi$ denotes the canonical projection from $\Omega$ into $\mathscr{R}$. A plaquette (link) is called horizontal or parallel to $\mathscr{R}$ if its projection is again a plaquette (link). A plaquette in $\mathscr{R}$ is called boundary plaquette if it contains a link in $\partial \mathscr{R}$. We say $P \in \Omega_{P}$ projects into the plaquette $P^{\prime}$ in $\mathscr{R}$ if $\pi(P)$ is equal to $P^{\prime}$ or a link or corner in $P^{\prime}$. Finally $\left(P_{1}, \ldots, P_{n}\right)$ is called a (self-avoiding) path of plaquettes if $P_{i}$ and $P_{i+1}$ are connected for $i=1, \ldots, n-1$ (and if the plaquettes $P_{1}, \ldots, P_{n}$ are pairwise distinct). The links common to $P_{i}, P_{i+1}(i=1, \ldots, n-1)$ are called the links in $\left(P_{1}, \ldots, P_{n}\right)$.

Since an exterior defect network $E$ can as well be described by $\varepsilon$ according to (5.7), we refer to $\varepsilon$ by a slight abuse of notation as the defect network $\varepsilon$ in the sequel.

Definition 6.1. We fix a defect network $\varepsilon$ with defect surface $\mathscr{E}$. A plaquette $P$ is called ceiling plaquette if $P$ is a horizontal plaquette in $\mathscr{E}$ and there is no other plaquette in $\mathscr{E}$ which projects into $\pi(P)$. The set of ceiling plaquettes is denoted $\mathscr{C}$. All other plaquettes in $\mathscr{E}$ are called wall plaquettes, the set of wall plaquettes is denoted $\mathscr{W}$. A horizontal wall plaquette $P$ is called quasi-ceiling plaquette if there is no other plaquette $P^{\prime}$ in $\mathscr{E}$, such that $\pi(P)=\pi\left(P^{\prime}\right)$, otherwise it is called proper wall plaquette (write $P \in \mathscr{W}_{0} \subset \mathscr{W}$ ). A function $\omega$ from $\Omega_{P}^{\infty}$ into $G$ is called ceiling respectively wall if its support is a connectivity component of $\mathscr{C}$ respectively $\mathscr{W}$ and if $\omega(P)=\varepsilon(P)$ for all plaquettes in the support of $\omega$. From the definition of $\mathscr{E}$ in Sect. 5, namely from (5.6), it is clear that the boundary plaquettes are ceiling plaquettes. The ceiling with support containing these plaquettes is called the regular ceiling.

Remark. If $P$ is a ceiling or quasi-ceiling plaquette, then $\varepsilon(P)=g_{0}$.

Proof. Choose a self-avoiding path $\left(P_{1}, \ldots, P_{n}\right)$ in $\mathscr{R}$, such that $P_{1}$ is a boundary plaquette (which implies $\left.\varepsilon\left(P_{1}\right)=g_{0}\right)$ and $P_{n}=\pi(P)$. Let $T_{i}$ be the set of plaquettes in $\mathscr{E}$ projecting onto $P_{i}$ and $\ell_{i}$ the link common to $P_{i}$ and $P_{i+1}$. We now apply the Bianchi-identity - which for all links $\ell$ not in $\partial \mathscr{R}$ reads

$$
\prod_{P: \ell \in \partial P} \varepsilon(P)=\mathbb{1},
$$

to the links $\ell$ with projection $\pi(\ell)=\ell_{i}$, and obtain

$$
\prod_{P \in T_{i}} \varepsilon(P)=\prod_{P \in T_{i+1}} \varepsilon(P) .
$$

But because $P_{1}$ and $P$ are ceiling or quasi-ceiling plaquettes, this implies

$$
\varepsilon(P)=\varepsilon\left(P_{1}\right)=g_{0} .
$$

We now state a lemma which reduces the study of the connectivity properties of $\mathscr{C}$ respectively $\mathscr{W}$ to that of their projections. This will make it possible to describe a defect network $\varepsilon$ by its walls, more precisely by its standard walls, see Definition 6.6 and Lemma 6.7 below.

Lemma 6.2. i) The projection $\pi(W)$ of a connectivity component $W$ of $\mathscr{W}$ is a connected set of points, links and plaquettes in $\mathscr{R}$. 
ii) The projections $\pi\left(W_{1}\right), \pi\left(W_{2}\right)$ of two distinct connectivity components of $\mathscr{W}$ are disconnected.

iii) The projection $\pi(C)$ of a connectivity component $C$ of $\mathscr{C}$ is a connected set of plaquettes in $\mathscr{R}_{P}$.

iv) The projections $\pi\left(C_{1}\right), \pi\left(C_{2}\right)$ of two distinct connectivity components of $\mathscr{C}$ are disconnected.

The proof is based on the following Lemmas 6.3, 6.4, and 6.5.

Lemma 6.3. Let $\left(\tilde{P}_{1}, \ldots, \widetilde{P}_{n}\right)$ be a self-avoiding path of plaquettes in $\mathscr{R}_{P}$, such that $\tilde{P}_{1}$ and $\tilde{P}_{n}$ are projections of ceiling or quasi-ceiling plaquettes: $\tilde{P}_{1}=\pi(P), \widetilde{P}_{n}=\pi\left(P^{\prime}\right)$. Then there is a path $\left(P_{1}, \ldots, P_{m}\right)$ of plaquettes in $\mathscr{E}$, such that the links in $\left(P_{1}, \ldots, P_{m}\right)$ are horizontal and

i) $P_{1}=P, P_{m}=P^{\prime}$.

ii) For any plaquette $P_{i}$ in $\left(P_{1}, \ldots, P_{m}\right), \pi\left(P_{i}\right)$ is a link or plaquette in $\left(\widetilde{P}_{1}, \ldots, \widetilde{P}_{n}\right)$.

iii) For any plaquette $\widetilde{P}_{i}$ in $\left(\tilde{P}_{1}, \ldots, \widetilde{P}_{n}\right)$ there is at least one plaquette $P_{j}$ in $\left(P_{1}, \ldots, P_{m}\right)$ such that $\pi\left(P_{j}\right)=\tilde{P}_{i}$.

Remark. In particular this implies that $\mathscr{E}$ has no holes in the sense that $\pi(\mathscr{E})$ contains all plaquettes in $\mathscr{R}_{P}$.

Proof of Lemma 6.3. Consider the set $T$ of plaquettes in $\mathscr{E}$ which project onto a plaquette or link in $\left(\widetilde{P}_{1}, \ldots, \widetilde{P}_{n}\right)$ and the set $L$ of links belonging to plaquettes in $T$ and projecting onto links in $\left(\widetilde{P}_{1}, \ldots, \widetilde{P}_{n}\right)$. Decompose $T$ into maximal sets $T_{1}, \ldots, T_{r}$ such that the plaquettes in $T_{i}$ are connected via links in $L$. Applying the Bianchiidentity in the form (6.1) to the links in $L$ it follows that the set $T_{k}$ containing $P$ also contains $P^{\prime}$. Because $T_{k}$ is connected via horizontal links it contains the desired path.

Lemma 6.4. Let $W$ be a connectivity component of $\pi(\mathscr{W})$.

i) If $W$ contains a point $x$, then it also contains at least one link $\ell$ in $\mathscr{R}$ such that $x \in \partial \ell$.

ii) If $W$ contains a point $x$ or a link $\ell$, it also contains all plaquettes containing $\ell$ respectively $x$ as link or corner.

Proof. i) Let $P$ be a plaquette in $\mathscr{E}$ projecting into $x$. Choose a path $\left(P_{1}, \ldots, P_{n}\right)$ in $\mathscr{E}$ from $P=P_{1}$ to some boundary plaquette $P_{n}$. Let $P_{k}$ be the first plaquette in the path which contains a horizontal link. Then $\pi\left(P_{k}\right)$ is the desired link in $\mathscr{R}$.

ii) For a link $\ell$ is clear, because a plaquette in $\mathscr{R}$ containing $\ell$ is always the projection of a wall plaquette. For a point $x$ in $W$ we use i) to deduce the existence of a link $\ell$ in $W$ with $x \in \partial \ell$. Let $P_{1}, \ldots, P_{4}$ be the four plaquettes containing $x$. The set $\left\{x, \ell, P_{1}, \ldots, P_{4}\right\}$ is a connected set in $\pi(\mathscr{W})$ which settles ii).

Lemma 6.5. Let $P_{1}$ be the projection of a ceiling plaquette and $P_{2}$ be the projection of a horizontal wall plaquette. If $P_{1}$ and $P_{2}$ share a common link, then $P_{2}$ is the projection of a quasi-ceiling plaquette and the corresponding plaquette $\widetilde{P}_{1}, \widetilde{P}_{2}$ in $\mathscr{E}$ are connected.

Proof. Let $\ell_{0}$ be the link common to $P_{1}$ and $P_{2}$, let $T_{1}$ respectively $T_{2}$ be the set of plaquettes in $\mathscr{E}$ which project onto $P_{1}$ respectively $P_{2}$, let $T$ be the set of plaquettes 
which project onto $\ell_{0}$. Since $P_{1}$ is ceiling plaquette, $T$ is empty and $T_{1}$ contains only one plaquette $\widetilde{P}_{1}$. Let $L$ be the set of links in plaquettes in $T$, which project onto $\ell_{0}$. Applying the Bianchi-identity in the form (6.1) to the links in $L$ we get that any such link $\ell$ must be common to at least two plaquettes in $T_{1} \cup T \cup T_{2}=T_{1} \cup T_{2}$. Since the projections of two plaquettes which are horizontal and contain a common link cannot be the same, it follows that any link in $L$ is common to exactly one plaquette in $T_{1}$ and one in $T_{2}$. This implies the lemma.

Proof of Lemma 6.2. i) and iii) are evident.

ii) We first prove that any connectivity component $W$ of $\mathscr{W}$ contains a quasiceiling plaquette. Let $P_{1}$ be a plaquette in $W$ and $\left(P_{1}, \ldots, P_{n}\right)$ a path in $\mathscr{E}$ from $P_{1}$ to some boundary plaquette $P_{n}$. Let $P_{k}$ be the first ceiling plaquette in $\left(P_{1}, \ldots, P_{n}\right)$. It follows that $P_{k-1}$ is a horizontal plaquette in $W$. Applying Lemma 6.5 to the projections of $P_{k}$ and $P_{k-1}$ we get that $P_{k-1}$ is a quasi-ceiling plaquette. ii) is now an easy consequence of Lemmas 6.3 and 6.4: let $P_{1}$ respectively $P_{2}$ be quasi-ceiling plaquettes in $W_{1}$ respectively $W_{2}$. If $\pi\left(W_{1}\right)$ and $\pi\left(W_{2}\right)$ are connected we can find a self-avoiding path from $\pi\left(P_{1}\right)$ to $\pi\left(P_{2}\right)$ in $\pi\left(W_{1}\right) \cup \pi\left(W_{2}\right)$ due to Lemma 6.4. Applying Lemma 6.3 to this path we get a path of wall plaquettes from $P_{1}$ to $P_{2}$. This is in contradiction to the assumption that $W_{1}$ and $W_{2}$ are different connectivity components of $\mathscr{W}$.

It remains to prove iv). But this is by now evident (use Lemma 6.3!).

We are now ready to reduce the study of a defect network $\varepsilon$ to the study of its walls. Consider a single wall $w$ with support $W$. Let $C$ be the connectivity component of $\mathscr{R}_{P} \backslash \pi(W)$ that contains the boundary plaquettes. We call $C$ the exterior of $\pi(W)$.

There is exactly one connectivity component of $\pi(\mathscr{C})=\mathscr{R}_{P} \backslash \pi(\mathscr{W})$ that connects to $\pi(W)$ and lies in the exterior of $\pi(W)$. The support of the ceiling that projects into this connectivity component is called the base of the wall $w$.

The height of a ceiling $c$ is the height of the plaquettes in the support of $c$, the height of a wall $w$ is the height of the plaquettes in its base. The drift $\Delta(w)$ of a wall $w$ is the translate $t_{-h}(w)$, where $h$ is the height of $w$.

Definition 6.6. A standard wall $w$ is a function from $\Omega_{P}^{\infty}$ into $G$ such that there is a defect network having $w$ as its unique wall. Two standard walls are called compatible if the projections of their supports are disconnected. The activity of a standard wall $w$ with support $W$ is defined as

$$
z(w)=\left(\prod_{P \in \Omega_{P}^{\infty}} e^{-s(w(P))}\right) e^{|\pi(W)| s\left(g_{0}\right)},
$$

where $|\pi(W)|$ denotes the number of plaquettes in $\pi(W)$. The size $|w|$ of $w$ is the number of plaquettes in $W:|w| \equiv|W|$.

Lemma 6.7. i) Let $\varepsilon$ be a defect network with walls $w_{1}, \ldots, w_{n}$. Then $\left\{\Delta\left(w_{1}\right), \ldots\right.$, $\left.\Delta\left(w_{n}\right)\right\}$ is a set of pairwise compatible standard walls.

ii) Let $\left\{w_{1}, \ldots, w_{n}\right\}$ be a set of pairwise compatible standard walls. Then there is exactly one defect network $\varepsilon$ with walls $v_{1}, \ldots, v_{n}$ such that $\Delta\left(v_{i}\right)=w_{i}$ for $i=1, \ldots, n$.

Proof. i) We first consider one of the walls, call it $w$, and show that $\Delta(w)$ is a standard wall. Let $W$ be the support of $w$ and $C_{1}, \ldots, C_{m}$ the connectivity 
components of $\mathscr{R}_{P} \mid \pi(W)$. Due to Lemmas 6.4 and $6.5 \pi(W)$ contains a plaquette $P_{k}$ which shares a link with a plaquette in $C_{k}(k=1, \ldots, m)$. Let $h_{k}$ be the height of the quasi-ceiling plaquette in $\mathscr{E}$ projecting onto $P_{k}$ and define $v_{k}=h_{k}-h_{0}$, where $h_{0}$ is the height of $w$. We now define a function $\varepsilon^{\prime}$ from $\Omega_{P}^{\infty}$ to $G$ :

$$
\varepsilon^{\prime}(P)=(\Delta(w))(P) \prod_{i=1}^{n} c_{i}(P),
$$

where $c_{i}$ is equal to $g_{0}$ on $t_{v_{i}}\left(C_{i}\right)$ and $\mathbb{1}$ anywhere else. We claim that $\varepsilon^{\prime}$ is a defect network with unique wall $\Delta(w)$. Since by construction the support of $\varepsilon^{\prime}$ is connected we only have to show the Bianchi-identity (6.1) for all links which are not in $\partial \mathscr{R}$. The only "critical" links are the links $\ell$ common to a plaquette $P$ in the support of $\Delta(w)$ and a plaquette $P^{\prime}$ in the support of some $c_{i}$. But due to Lemma 6.5 and the remark after Definition $6.1 P$ is the only plaquette in the support of $\Delta(w)$ containing the link in question and $(\Delta(w))(P)=g_{0}$. This proves the Bianchiidentity for the critical links. Note that due to the Bianchi identity the above construction of a defect network $\varepsilon^{\prime}$ with unique wall $\Delta(w)$ is the only possible one, i. a. $\varepsilon^{\prime}$ is unique, given $\Delta(w)$.

Up to now we have shown that $\left\{\Delta\left(w_{1}\right), \ldots, \Delta\left(w_{n}\right)\right\}$ is a set of standard walls. Compatibility and therefore i) follows from Lemma 6.2.

ii) Will be done by induction. Suppose $\varepsilon$ is a defect network with walls $w_{1}, \ldots, w_{n}$ and let $w_{0}$ be a standard wall compatible with $\Delta\left(w_{i}\right)$ for $i=1, \ldots, n$. We have to construct a defect network $\varepsilon^{\prime}$ with walls $w_{0}^{\prime}, \ldots, w_{n}^{\prime}$ such that $w_{0}=\Delta\left(w_{0}^{\prime}\right)$, $\Delta\left(w_{i}\right)=\Delta\left(w_{i}^{\prime}\right)$ for $i=1, \ldots, n$.

Denote the support of $w_{0}$ by $W_{0}$ and let $P_{0}$ be a plaquette in $\pi\left(W_{0}\right)$ that shares a link with a plaquette in the exterior of $\pi\left(W_{0}\right) . h_{0}$ is then the height of the ceiling plaquette in $\mathscr{E}$ that projects onto $P_{0} . w_{0}^{\prime}$ is defined as $w_{0}^{\prime}=t_{h_{0}}\left(w_{0}\right)$.

Let $\varepsilon_{0}$ be the defect network having $w_{0}$ as its unique wall, let $C_{1}, \ldots, C_{m}$ be the connectivity components of $\mathscr{R}_{P} \backslash \pi\left(W_{0}\right)$. Let $P$ be a plaquette in $\pi\left(W_{0}\right)$ which shares a link with a plaquette in $C_{k}$ and define $h_{k}$ as the height of the quasi-ceiling plaquette in $\mathscr{E}_{0}$ that projects onto $P(k=1, \ldots, m)$. Consider a wall $w_{i}$ with support $W_{i}$ projecting into $C_{k} . w_{i}^{\prime}$ is then defined as $w_{i}^{\prime}=t_{h_{k}}\left(w_{i}\right)$. Note that $w_{i}$ is not translated if $h_{k}$ is zero, e.g. if $C_{k}$ is the exterior of $\pi\left(W_{0}\right)$.

It is now clear how to define $\varepsilon^{\prime}$ : consider the ceiling plaquettes $P_{1}, \ldots, P_{N}$ of $\mathscr{E}$ not projecting into $\pi\left(W_{0}\right)$. Let $h\left(P_{i}\right)=h_{k}$ if $P_{i}$ projects into $C_{k}$. $\varepsilon^{\prime}$ is the function from $\Omega_{P}^{\infty}$ into $G$ that is $g_{0}$ on $P_{i}^{\prime}=t_{h\left(P_{i}\right)}\left(P_{i}\right)$ for $i=1, \ldots, N, w_{i}^{\prime}(P)$ if $P$ lies in the support of $w_{i}^{\prime}, i=0, \ldots, n$ and $\mathbb{1}$ everywhere else. As in i) one proves that $\varepsilon^{\prime}$ is really a defect network with walls $w_{0}^{\prime}, \ldots, w_{n}^{\prime}$. Again from the construction it is clear that $\varepsilon^{\prime}$ is unique.

Lemma 6.7 reduces the study of the defect network to the study of compatible standard walls. Note that $z(w)$ is defined in such a way that

$$
z(E)=\prod_{i=1}^{n} z\left(w_{i}\right) \exp \left\{-s\left(g_{0}\right) L_{3} L_{4}\right\}
$$

if $E$ is the defect network corresponding to the standard walls $w_{1}, \ldots, w_{n}$. We now have to bound the probability of large standard walls. This would be trivial if in the probability of a given defect network (see Lemma 3.3) there would be no 
interaction part $V_{A}(E)$. To deal with this interaction we use the "renormalization" trick of Dobrushin [3].

For a standard wall $w$ with support $W$ let $U(w)$ be the collection of points in the plane $z_{1}=z_{2}=-1 / 2$ which are points in $\pi(W)$ or centers of plaquettes and links in $\pi(W)$. For $u \in U(w) n(u)$ denotes the number of plaquettes in $W$ which have a center projecting onto $u$. Two standard walls $w_{1}, w_{2}$ with supports $W_{1}, W_{2}$ are called distant if for all points $u_{1} \in U\left(w_{1}\right), u_{2} \in U\left(w_{2}\right)$

$$
\left|u_{1}-u_{2}\right|>\sqrt{n\left(u_{1}\right)}+\sqrt{n\left(u_{2}\right)}
$$

and close otherwise.

Definition 6.8. Let $G$ be a set of pairwise compatible standard walls. $G$ is called a group of walls, if for any two walls $w, w^{\prime}$ in $G$ one can find a sequence $\left(w_{1}, \ldots, w_{n}\right)$ in $G$ such that $w=w_{1}, w^{\prime}=w_{n}$, and $w_{i}, w_{i+1}$ are close for $i=1, \ldots, n-1$. Two groups $G_{1}, G_{2}$ of walls are called compatible if for any two walls $w_{1} \in G_{1}, w_{2} \in G_{2}, w_{1}$ and $w_{2}$ are compatible and distant. For a group of walls $G=\left\{w_{1}, \ldots, w_{n}\right\}$ one defines

$$
\begin{gathered}
z(G)=\prod_{i=1}^{n} z\left(w_{i}\right), \\
|G|=\sum_{i=1}^{n}\left|w_{i}\right|,
\end{gathered}
$$

and finally the union of the supports of $w_{1}, \ldots, w_{n}$ is called the support of $G$.

We now claim the existence of a function $g_{1}(b)$, going to zero with $b$ and depending only on the dimension and $K_{0}$, such that the following lemma holds.

Lemma 6.9. i) Let $\left\{G_{0}, \ldots, G_{n}\right\}$ be a set of pairwise compatible groups of walls. Let $E$ be the exterior defect network corresponding to $\left\{G_{1}, \ldots, G_{n}\right\}$ and $E^{\prime}$ the exterior defect network corresponding to $\left\{G_{0}, \ldots, G_{n}\right\}$. Then for $b>\log K_{0}+\log 2.93$

$$
p_{b}\left(E^{\prime}\right) \leqq z\left(G_{0}\right) e^{\left|G_{0}\right| g_{1}(b)} p_{b}(E) .
$$

ii) For $b>\log K_{0}+\log 2.93$ the probability $p(G)$ of a group $G$ of walls is bounded by

$$
z(G) e^{|G| g_{1}(b)} .
$$

Proof. ii) is an obvious consequence of i), using that $\sum_{E} p_{b}(E)=1$.

i) We apply the results of Sect. 3. Due to (6.3), (6.5), and Lemma 3.3

$$
p_{\underline{b}}\left(E^{\prime}\right)=p_{\underline{b}}(E) z\left(G_{0}\right) e^{-\Delta V},
$$

where

$$
|\Delta V|=\left|\sum_{P \in E} v(P, E, \Lambda)-\sum_{P^{\prime} \in E^{\prime}} v\left(P^{\prime}, E^{\prime}, \Lambda\right)\right| .
$$

Let $G_{0}$ contain the standard walls $w_{1}, \ldots, w_{m}$ with supports $W_{1}, \ldots, W_{m}$. Consider the construction of $\varepsilon^{\prime}$ from $G_{0}$ and $\varepsilon$ following the lines of the proof of Lemma 6.7. Let $\mathscr{E}$ be the support of $\varepsilon, \mathscr{E}$ ' that of $\varepsilon^{\prime}$. Then $\mathscr{E}$ ' is obtained from $\mathscr{E}$ as follows:

Subtract from $\mathscr{E}$ all the ceiling plaquettes projecting into $\pi(W)=\pi\left(W_{1}\right) \cup \ldots$ $\cup \pi\left(W_{m}\right)$. Translate each plaquette in the remaining set $\widetilde{\mathscr{E}}$ by a suitable vector $v(P)$. Add the plaquettes of $W_{1}, \ldots, W_{m}$, properly translated. 
If we denote the translate of $P$ by $P^{\prime}$ and apply Lemma 3.4 we get

$$
|\Delta V| \leqq g(b)\left\{\left|G_{0}\right|+|\pi(W)|\right\}+\sum_{P \in \tilde{\delta}}\left|v(* P, E, \Lambda)-v\left(* P^{\prime}, E^{\prime}, \Lambda\right)\right|,
$$

where $* P$ denote the dual of $P$. From the definition of $d(\ldots)$ in Sect. 3 and the definition of walls and ceilings, it follows that

$$
d\left(* P, E, \Lambda ; * P^{\prime}, E^{\prime}, \Lambda\right) \geqq r(P),
$$

where $r(P)$ denotes the distance of the center of $\pi(P)=\pi(\widetilde{P})$ to the set $\pi(W)$. Using Lemma 3.4 again and bounding $|\pi(W)|$ by $\left|G_{0}\right|$ we get

$$
|\Delta V| \leqq 2\left|G_{0}\right| g(b)+g(b-\alpha) \sum_{P \in \tilde{\mathscr{E}}} e^{-\alpha r(P)},
$$

where we defined $\alpha=\left(b-\log K_{0}-\log 2.93\right) / 2$. Let $U\left(G_{0}\right)$ be the union of the sets $U\left(w_{i}\right), i=1, \ldots, n$, let $U(\Omega)$ be the collection of points in the plane $z_{1}=z_{2}=-1 / 2$ which are points in $\pi\left(\Omega_{P}\right)$ or centers of links or plaquettes in $\pi\left(\Omega_{P}\right)$ and define for $u \in U(\Omega) r(u)$ as the distance from $u$ to the set $U\left(G_{0}\right)$. Finally let $\bar{U}=U(\Omega) \backslash U\left(G_{0}\right)$. Then

$$
\sum_{P \in \tilde{\mathscr{E}}} \exp (-\alpha r(P)) \leqq \sum_{u \in \bar{U}} n(u) e^{-\alpha r(u)},
$$

where $n(u)$ is the number of plaquettes $P$ in $\widetilde{\mathscr{E}}$ such that the center of $P$ projects onto $u$. Since all the standard walls corresponding to $E$ are distant from the walls $w_{1}, \ldots, w_{m}$ we get, using (6.5),

$$
\begin{aligned}
\sum_{P \in \tilde{E}} \exp (-\alpha r(P)) & \leqq \sum_{u \in \bar{U}}\left(1+r(u)^{2}\right) e^{-\alpha r(u)} \\
& \leqq \sum_{u_{1} \in \widetilde{U}} \sum_{u_{2} \in U\left(G_{0}\right)}\left(\left|u_{1}-u_{2}\right|^{2}+1\right) e^{-\alpha\left|u_{1}-u_{2}\right|} \\
& \leqq\left|U\left(G_{0}\right)\right| g_{2}(\alpha) \leqq\left|G_{0}\right| g_{2}(\alpha)
\end{aligned}
$$

with some function $g_{2}(\alpha)$ going to zero with $\alpha \rightarrow \infty$.

Putting everything together we obtain i) with

$$
g_{1}(b)=2 g(b)+g_{2}(\alpha(b)) g(b-\alpha(b)),
$$

which goes to zero with $b \rightarrow \infty$.

Lemma 6.10. Let $b_{0}, b$ be according to Sect. 1 . Let $b_{1}=2 b-b_{0}$. Then

$$
\sum_{G}^{\prime} z(G) \leqq e^{-b_{1} s / 3},
$$

where the sum goes over groups of walls with fixed support of size s.

Proof of Lemma 6.10. Due to Definition 6.8 it is enough to prove

$$
\sum_{w}^{\prime} z(w) \leqq e^{-b_{1}|W| / 3},
$$

where the sum goes over all standard walls with support $W$. Let $W_{0}$ be the set of quasi-ceiling plaquettes in $W$, let $W_{1}$ be the set of horizontal proper wall plaquettes in $W$ and let $W_{2}=W /\left(W_{0} \cup W_{1}\right)$. It is clear that $\pi\left(W_{2}\right)|=0,| \pi\left(W_{0}\right)|=| W_{0} \mid$ and $2\left|\pi\left(W_{1}\right)\right| \leqq\left|W_{1}\right|$, where $\left|\pi\left(W_{i}\right)\right|$ denotes the number of plaquettes in $\pi\left(W_{i}\right)$. 
Together with (6.2) and the remark after Definition 6.1 this gives

$$
z(w) \leqq \prod_{P \in W_{1} \cup W_{2}} e^{-s(w(P))} e^{\left|W_{1}\right| b_{0} / 2},
$$

yielding

$$
\sum_{w}^{\prime} z(w) \leqq \exp \left(\frac{1}{2} b_{0}\left|W_{1}\right|-b\left|W_{1}\right|-b\left|W_{2}\right|\right) .
$$

Let $P$ be a plaquette in $W_{0}$. By definition of a quasi-ceiling plaquette $\pi(P)$ contains at least one point or link which is the projection of a plaquette in $W_{2}$. Since a point (link) in $\pi\left(W_{2}\right)$ can be shared by maximal four (two) plaquettes in $\pi\left(W_{0}\right)$ it follows $\left|\pi\left(W_{0}\right)\right| \leqq 4\left|W_{2}\right|$. Using Lemma 6.4 this can be sharpened to $\left|W_{0}\right|=\left|\pi\left(W_{0}\right)\right| \leqq 2\left|W_{2}\right|$ yielding

$$
\left|W_{2}\right|=\frac{1}{3}\left|W_{2}\right|+\frac{2}{3}\left|W_{2}\right| \geqq \frac{1}{3}\left(\left|W_{2}\right|+\left|W_{0}\right|\right)
$$

Using $b \leqq b_{0}$, i.e. $b_{1} \leqq b$ we get (6.7).

With Lemmas 6.9 and 6.10 the proof of Lemma 5.3 reduces essentially to a combinatoric estimate, which is presented in the proof of Lemma 6.12 below, the last lemma of this section. To state the lemma we need

Definition 6.11. A standard wall $w$ with support $W$ is compatible with a plaquette $P$ in $\mathscr{R}_{P}$ if there is a path $\left(P_{1}, \ldots, P_{n}\right)$ in $\mathscr{R}_{P} \backslash \pi(W)$ such that $P_{1}=P$ and $P_{n}$ is a boundary plaquette. A group $G$ of walls is compatible with $P$ if all the walls in $G$ are compatible with $P$.

From this definition it is clear that for a fixed defect network with groups of walls $G_{1}, \ldots, G_{n} P \in \mathscr{R}_{P}$ is a ceiling plaquette if it is compatible with all the groups $G_{1}, \ldots, G_{n}$.

Lemma 6.12. There is a constant $K_{2}$ depending only on the dimensiond such that for $b>\log K_{0}+\log 2.93$,

$$
\sum_{G}^{\prime} p(G) \leqq\left(K_{2} e^{g_{1}(b)} e^{-b_{1} / 3}\right)^{s},
$$

where the sum goes over groups of walls $G$ of size $|G|=s$ which are incompatible with $P$.

Proof. Consider a group of walls $G$ with support $T$. Note that $U(G)$, as well as $n(u)$ for $u \in U(G)$ depend only on $T$; we therefore write $U(T)$ instead of $U(G)$. Let $X$ be the set of points which are centers of plaquettes in $\mathscr{R}_{P}$ and assign to each point $u \in U(T)$ the collection $X_{u}$ of points $x$ in $X$ such that $|x-u|^{2}<n(u)$. Let $\tilde{T}$ be the set of plaquettes and points

$$
\tilde{T}=T \cup \bigcup_{u \in U(T)} X_{u}
$$

Because $G$ is a group of walls it follows that $\tilde{T}$ is a connected set with the evident definition of connectivity. Further $\tilde{T}$ uniquely determines $T$ and

$$
|\tilde{T}| \leqq|T|+\sum_{u \in U(T)}\left|X_{u}\right| \leqq|T|+\pi \sum_{u \in U(T)} n(u) \leqq 5|T|,
$$

where $|\widetilde{T}|$ denotes the number of plaquettes and points in $\widetilde{T}$. 
Next we use the fact that $G$ is incompatible with $P$. Let $w$ be a standard wall with support $W \subset T$ that is incompatible with $P$. Let $\left(0,0, x_{3}, x_{4}\right)$ be the position of $P$ in $\mathscr{R}_{P}$ and define a path $\left(P_{1}, \ldots, P_{n}\right)$ in $\mathscr{R}_{P}$ such that $P_{i}$ has position $\left(0,0, x_{3}+i-1, x_{4}\right)$. If we choose $n=|T| \geqq|\pi(W)|$ it is geometrically obvious that $\pi(W)$ has a plaquette in common with $M=\left\{P_{1}, \ldots, P_{n}\right\}$. Because $w$ is a standard wall, also $W$ has a (quasi-ceiling) plaquette in common with $M$.

We now can bound the number of possible supports of groups of walls with size $s$, incompatible with $P$ by the number of connected set $\tilde{T}$ of size $\tilde{s} \leqq 5 s$ having nonempty intersection with $M$. As usual this number can be bounded by

$$
\sum_{\tilde{s} \leqq 5 s}|M| C_{1}^{\tilde{s}} \leqq 5 s^{2} C_{1}^{5 s}
$$

with some constant $C_{1}$ depending only on the dimension $d$. Together with Lemma 6.9 and 6.10 we get Lemma 6.12 .

Proof of Lemma 5.3. This is now almost obvious.

i) is already proven, see the remark after Definition 6.1 .

ii) Due to the remark after Definition 6.11 and Lemma 6.12 we can bound the probability that a given plaquette in $\mathscr{R}_{P}$ is not a ceiling plaquette by

$$
\sum_{s \geqq 1} \varrho(J)^{s}=\varrho(J)(1-\varrho(J))^{-1}
$$

with

$$
\varrho(J)=K_{2} e^{g_{1}(b)} e^{-b_{1} / 3} .
$$

To complete the proof of Lemma 5.3 we have to show that $b_{1} \rightarrow \infty$ with $J \rightarrow \infty$. For finite gauge groups with cardinality $N$ it is evident from (1.4), (1.5) that $b \geqq b_{0}-\log (N-1)$ and therefore $b_{1}=2 b-b_{0}=0(b)$ which goes to infinity with $J \rightarrow \infty$.

Remark. This is the only step in the proof of Lemma 5.3 respectively Theorem 5.1 where besides discreteness of the abelian group $G$ we used finiteness of $G$. Therefore Lemma 5.3 and Theorem 5.1 remain true for an arbitrary discrete abelian group $G$ as long as $2 b-b_{0}$ goes to infinity with $J$.

\section{Concluding Remarks}

Here we want to summarize our results and to discuss possible generalizations. We first consider the results of Sect. 5 respectively 6. In four dimensions we have constructed a non-translation invariant equilibrium state for the weak coupling lattice gauge theory with discrete abelian group (Theorem 5.1). We first introduced b.c. which force a magnetic flux sheet into the system and then showed that it remains localized in the thermodynamic limit. It is clear that this does not generalize to the $U(1)$ theory, since there the magnetic flux will spread and in the thermodynamic limit translation invariance will be recovered.

To discuss other possible generalizations we use the well-known fact (see [5] for a discussion of the interplay between electric and magnetic properties in lattice gauge theories) that a localized magnetic flux implies spreading of the electric flux, 
which means deconfinement for electric charges in the sense of ' $t$ Hooft ([14], see also [15]). For the standard Wilson action this should be equivalent to deconfinement in the ordinary sense (see however [16] for a model where probably the 't Hooft string tension is zero, but not the usual one). Therefore a generalization of Theorem 5.1 can not work in a theory with confinement, at least if we do not consider models like that in [16].

But one might try to generalize the results of Sect. 5 respectively 6 in the high temperature, deconfinement phase of Yang-Mills theories. There however the time direction is periodic with period $L_{4}=(\varepsilon T)^{-1}$, where $T$ is the temperature and $\varepsilon$ the lattice spacing (which we fixed to 1). Then the magnetic flux sheet becomes a ribbon, and this is essentially a one dimensional object. This probably fluctuates so much in the infinite volume limit that translation invariance is restored.

If we choose the 1-direction to be the time direction this objection does not apply. We then really expect the existence of a non-translation invariant state, but since it is no more time translation invariant, it is not clear if it has any physical interpretation.

Next we discuss the results of Sect. 2. We have shown uniqueness of the translation invariant equilibrium states in lattice gauge theories with finite abelian group $G$, provided we are in the weak coupling regime.

We first consider the somewhat technical question whether the result also holds for finite non-abelian groups. Since we made extensive use of the Bianchiidentity from the beginning one might be worried whether the proof of Theorem 2.1 goes through for non-abelian groups. In particular one could ask how to define defect networks and polymers, and how to do the cluster expansion. I do not want to enter into details here, but at least for me it is not evident that the straightforward generalization of the abelian weak coupling cluster expansion works in the non-abelian case, as it was claimed in [5]. I have no doubts however that also for non-abelian finite gauge groups one can write down a converging cluster expansion (and apart from technical details it will be that presented in [5]). It is clear that then the methods of Sect. 2 could be applied to prove uniqueness of the translation invariant equilibrium states for weak couplings.

Next consider infinite discrete abelian groups, e.g. the integers $\mathbb{Z}$. We note that the strategy of Sect. 2 does not go through: in order to prove that the probability of large (exterior) defect networks is small in the sense of Lemma 2.3 we had to use [see (3.3)] that the interaction $s(\cdot)$ is bounded.

This is not only a technical problem. Indeed for the three dimensional lattice gauge theory with gauge group $\mathbb{Z}$ and interaction $s(z)=g^{-2} z^{2}$ one can choose b.c. which force large defect networks into the volume and lead to non-standard translation invariant equilibrium states.

Consider a finite box $\Lambda=[1, L]^{3} C \mathbb{Z}^{3}$. Call the 3-component of a point in $\Lambda$ its height $h$. Denote the two faces of $\partial \Lambda$ which are orthogonal to the 1-direction by $S_{1}$ respectively $S_{L}$. We introduce the following b.c. on $\partial \Lambda$ : define $z_{x y}=h$ if $\langle x y\rangle$ is a positively oriented link in $\partial \Lambda$ which is parallel to the 2-direction and has height $h$, and $z_{x y}=0$ for the remaining (positively oriented) links in $\partial \Lambda$. Then the plaquette variables $z_{P}$ for plaquettes $P$ in $S_{1}$ respectively $S_{L}$ will be one, and for the other plaquettes in $\partial \Lambda$ they will be zero. 
The defect network with minimal energy will be that for which all plaquettes parallel to $S_{1}$ are frustrated (the corresponding plaquette variable will be $z_{P}=1$ ) and all the other ones are not $\left(z_{P}=0\right)$. It is plausible that the thermodynamic limit of the finite volume states with these b.c. exists and (eventually after averaging) is translation invariant. In the thermodynamic limit the fluctuations away from the defect network with minimal energy (the quantum fluctuations) should not influence the expectation values of $z_{P}$ (but they will influence those of $z_{P}^{2}$ ). Indeed from (the plausible) translation invariance and the conservation of the magnetic flux it follows that $\left\langle z_{P}\right\rangle=1$ for plaquettes orthogonal to the 1-direction and $\left\langle z_{P}\right\rangle=0$ for the other ones. $\left\langle z_{P}^{2}\right\rangle-\left\langle z_{P}\right\rangle^{2}$ on the other hand will be $o\left(g^{2}\right)$. Note that this equilibrium state, while translation invariant, breaks the (restricted) rotation symmetry of the lattice. Clearly the corresponding equilibrium state also exists in four dimensions.

It is interesting to replace the group $\mathbb{Z}$ by $\mathbb{R}$ [the "non-compact $U(1)$ "] and go to the continuum limit. For the b.c. analogous to those described above the classical field configuration (i.e. that with minimal energy) will be a constant magnetic field in the 1-direction. Again the quantum fluctuations of the field should not influence the expectation value, and even the introduction of matter fields should not influence the result, since the magnetic flux is conserved in a theory without monopoles.

Note that for these b.c. the compact formulation seems not to be a good approximation for the non-compact one, because the fields are no more close to zero. To see this consider the U(1) Wilson theory on a lattice with the b.c. obtained from the above ones by replacing $z_{x y}$ by $g_{x y}=\exp i$ a $z_{x y}$, where the constant a depends on the lattice spacing $\varepsilon$ which we set to one before; again the field with constant magnetic field is a possible field configuration; but since now the interaction $s(\cdot)$ is bounded, there will be field configurations with smaller energy: simply take the fields $g_{x y}=\mathbb{1}$ everywhere inside the box. The energy will be proportional to $|\partial \Lambda|$, whereas for the constant magnetic field it is proportional to $|\Lambda|$. So the compact version seems to reproduce the standard translation and rotation invariant equilibrium state, at least if the thermodynamic limit is taken before the continuum limit, whereas the non-compact version does not.

We finally point out that the existence of equilibrium states describing a constant magnetic field in non-compact QED is not so astonishing. They are realized for small space-time regions in any laboratory.

\section{Appendix: Convergence of Cluster Expansions}

In this appendix I present a corrected version of the proofs in [5] respectively [13] concerning cluster expansion convergence. Recall the following definitions from Sect. 3: a function $\gamma$ from the positively oriented plaquettes in $\mathbb{Z}^{d}$ into the gauge group $G$ which satisfies the Bianchi-identity is called an ordinary polymer, if its support is finite, non-empty and coconnected. The number of plaquettes in the support of $\gamma$ is called the size $|\gamma|$ of $\gamma$. An ordinary polymer $\gamma$ is called compatible with a set $T$ of plaquettes (another polymer $\gamma^{\prime}$ ) if the support of $\gamma$ coconnects to 
none of the plaquettes in $T$ (in the support of $\gamma^{\prime}$ ). Finally the activity $z(\gamma)$ of a polymer $\gamma$ is defined as

$$
z(\gamma) \equiv \prod_{P} e^{-s(\gamma(P))}
$$

where the interaction $s$ is a non-negative function on $G$ such that $s(g)=0$ only for $g=\mathbb{1}$. We recall the definition

$$
e^{-b} \equiv \sum_{g \neq \mathbb{1}} e^{-s(g)},
$$

and the fact that the number of coconnected sets of plaquettes in $\mathbb{Z}^{d}$ with fixed size $s$, such that one of the plaquettes coconnects to a given plaquette $P_{0}$ is bounded by $K_{0}^{s}$ with some constant $K_{0}>0$ (Lemma 1.5). We set

$$
\varepsilon=K_{0} e^{-b}
$$

and state the simple

Lemma A.1. Let $T$ be a set of plaquettes, $s$ be an integer $s>0$. Then

$$
\sum_{\gamma}^{\prime}|z(\gamma)| \leqq|T| \varepsilon^{s}
$$

where the sum goes over ordinary polymers $\gamma$ of fixed size s which are incompatible with $T$.

Proof. We first bound the sum over $\gamma$ 's with fixed support $T_{0}$ by $\exp \left(-b\left|T_{0}\right|\right)$ and then apply Lemma 1.5 to the number of possible supports for polymers arising in $\Sigma^{\prime}$. This yields the lemma.

To state the next lemma, which was originally proven by Rota in [12], we recall the following definition from Sect. 3: let $\gamma_{1}, \ldots, \gamma_{n}$ be a set of polymers and define $G\left(\left\{\gamma_{i}\right\}\right)$ to be the graph with vertices $\gamma_{1}, \ldots, \gamma_{n}$ and a line between $\gamma_{i}, \gamma_{j}(i \neq j)$ whenever $\gamma_{i}$ and $\gamma_{j}$ are incompatible. $a\left(\left\{\gamma_{i}\right\}\right)$ is then defined by

$$
a\left(\left\{\gamma_{i}\right\}\right)=\sum_{C \subset G\left(\left\{\gamma_{i}\right\}\right)}(-1)^{\ell(C)}
$$

where the sum is over connected subgraphs of $G\left(\left\{\gamma_{i}\right\}\right)$ and $\ell(C)$ is the number of lines in $C$.

Lemma A.2. $\left|a\left(\left\{\gamma_{i}\right\}\right)\right|$ can be bounded by the number of trees on $G\left(\left\{\gamma_{i}\right\}\right)$.

Proof. See for example [5] for a readable proof.

In the next lemma, which is based on a paper by Cammarota [13], $r_{0}$ denotes the minimal polymer size, i.e.

$$
r_{0}=2 d-2 \geqq 4
$$

since we supposed $d>3$.

Lemma A.3. Let $T$ be a set of plaquettes. Then

$$
\sum_{n=1}^{\infty} \sum_{\gamma_{1}, \ldots, \gamma_{n}}^{\prime} \frac{\left|a\left(\left\{\gamma_{i}\right\}\right)\right|}{n !} \prod_{i=1}^{n}\left|z\left(\gamma_{i}\right)\right| \leqq|T| \frac{\varepsilon^{r_{0}}}{1-\varepsilon}\left(1-\frac{8 r_{0} \varepsilon^{r_{0}}}{(1-\varepsilon)^{2}}\right)^{-1}
$$


where the sum $\sum^{\prime}$ goes over $\gamma_{1}, \ldots, \gamma_{n}$ for which at least one $\gamma_{i}$ is incompatible with a plaquette in $T$. The right-hand side has to be interpreted as infinity if

$$
\frac{8 r_{0} \varepsilon^{r_{0}}}{(1-\varepsilon)^{2}} \geqq 1
$$

Remark. i) Note that the right-hand side of (A.3) is finite if

$$
\begin{array}{lll}
1 / \varepsilon>2.93 & \text { for } & r_{0} \geqq 4(d \geqq 3), \\
1 / \varepsilon>2.31 & \text { for } & r_{0} \geqq 6(d \geqq 4)
\end{array}
$$

ii) Absolute convergence of the expansions (3.11) and (3.12) follows by setting $T=\Lambda_{P}$ and noting that

$$
\left|z_{E}(\gamma)\right| \leqq\left|z_{\Lambda}(\gamma)\right| \leqq|z(\gamma)|
$$

Proof. We use Lemma A.2 to bound the left-hand side of (A.3) by

$$
\sum_{n=1}^{\infty} \frac{1}{n !} \sum_{\gamma_{1}, \ldots, \gamma_{n}}^{\prime} \sum_{T_{n} \subset G\left(\left\{\gamma_{\gamma}\right\}\right)} \prod_{i=1}^{n}\left|z\left(\gamma_{i}\right)\right|
$$

where the last sum is the sum over trees $T_{n}$ contained in $G\left(\left\{\gamma_{i}\right\}\right)$. Next we change the order of summation and get

$$
\sum_{n=1}^{\infty} \frac{1}{n !} \sum_{T_{n}} \omega\left(T_{n}\right)
$$

where the sum over $T_{n}$ is now over all trees on $n$ vertices and

$$
\omega\left(T_{n}\right)=\sum_{\substack{\gamma_{1}, \ldots, \gamma_{n}: \\ T_{n} \subset G\left(\left\{\gamma_{i}\right)\right.}}^{\prime} \prod_{i=1}^{n}\left|z\left(\gamma_{i}\right)\right|
$$

One may bound the sum $\sum^{\prime}$ by assuming that $\gamma_{1}$ is incompatible with $T$ and multiplying by $n$. Inserting Lemma A.1 we get

$$
\omega\left(T_{n}\right) \leqq|T| n \sum_{s_{1}, \ldots, s_{n} \geqq r_{0}} s_{1} \prod_{i=1}^{n} s_{i}^{d_{i}-1} \varepsilon^{s_{i}}
$$

where $d_{i}$ is the number of lines in $T_{n}$ incident at the vertex $i$. We use Lemma 3.1 to bound $\omega\left(T_{n}\right)$ by

$$
\begin{aligned}
& n|T| \varepsilon^{n r_{0}} d_{1} ! \frac{1+d_{1} r_{0}^{d_{1}}}{(1-\varepsilon)^{d_{1}+1}} \prod_{i=2}^{n}\left(d_{i}-1\right) ! \frac{1+\left(d_{i}-1\right) r_{0}^{d_{i}-1}}{(1-\varepsilon)^{d_{i}}} \\
& \quad \leqq n|T| \varepsilon^{n r_{0}} \frac{r_{0}\left(1+d_{1}\right)}{(1-\varepsilon)} \prod_{i=1}^{n} \frac{d_{i} ! r_{0}^{d_{i}-1}}{(1-\varepsilon)^{d_{i}}} .
\end{aligned}
$$

Next we note that $\sum d_{i}=2 n-2$ and bound

$$
\left(1+d_{1}\right) \prod_{i=2}^{n} d_{i} \leqq \max _{\substack{q_{i} \geq 0 \\ \Sigma q_{i}=2 n-1}} \prod_{i=1}^{n} q_{i} \leqq\left(\frac{2 n-1}{n}\right)^{n} \leqq 2^{n} .
$$


Using again $\sum d_{i}=2 n-2$ and $d_{1} \leqq n-1$ we get

$$
\omega\left(T_{n}\right) \leqq|T| \frac{2 \varepsilon^{r_{0}}}{1-\varepsilon}\left(\frac{2 r_{0} \varepsilon^{r_{0}}}{(1-\varepsilon)^{2}}\right)^{n-1} \times n(n-1) \prod_{i=1}^{n}\left(d_{i}-1\right) !
$$

By Cayley's formula the number of trees on $n$ vertices with fixed $d_{i}$ 's is

$$
\frac{(n-2) !}{\left(d_{1}-1\right) ! \ldots\left(d_{n}-1\right) !}
$$

and by a simple combinatoric argument the number of sequences $d_{1}, \ldots, d_{n}$ with $\sum d_{i}=2 n-2$ is

$$
\left(\begin{array}{c}
2 n-3 \\
n-1
\end{array}\right) \leqq 2^{2 n-3}
$$

Putting everything together we get

$$
\begin{aligned}
\sum_{n \geqq 1} \frac{1}{n !} \sum_{T_{n}} \omega\left(T_{n}\right) & \leqq|T| \frac{\varepsilon^{r_{0}}}{(1-\varepsilon)} \sum_{n=1}^{\infty}\left(\frac{8 r_{0} \varepsilon^{r_{0}}}{(1-\varepsilon)^{2}}\right)^{n-1} \\
& =|T| \frac{\varepsilon^{r_{0}}}{(1-\varepsilon)}\left(1-\frac{8 r_{0} \varepsilon^{r_{0}}}{(1-\varepsilon)^{2}}\right)^{-1}
\end{aligned}
$$

which proves (A.3).

Remark. The proof is a little bit more involved than that one presented in [5] and [13], since we had to correct an error present in both references.

We finally prove absolute convergence for the cluster expansions (4.5) and (4.6). In the following let $N_{A}$ be the number of plaquettes in $\mathscr{A}$,

$$
N_{A}=|\mathscr{A}| \text {. }
$$

Lemma A.4. i) Let $\gamma_{A}$ be a polymer corresponding to $A, s$ an integer $s \geqq 0$. Then

$$
\sum_{\gamma}^{\prime}|z(\gamma)| \leqq\left(N_{A}+\left|\gamma_{A}\right|\right) \varepsilon^{s}
$$

where the sum goes over ordinary polymers $\gamma$ of fixed size s incompatible with $\gamma_{A}$.

ii)

$$
\sum_{\gamma_{A}}^{\prime}\left|z_{A}\left(\gamma_{A}\right)\right| \leqq\|A\|(1+s)^{N_{A}-1}
$$

where the sum goes over polymers $\gamma_{A}$ corresponding to $A$ of fixed size s.

Proof. i) is an evident consequence of Lemma A.1 since a polymer incompatible with $\gamma_{A}$ is incompatible with one of the plaquettes in $\mathscr{A}$ or in the support of $\gamma_{A}$.

To prove ii) we note that the support of a polymer corresponding to $A$ decomposes into coconnectivity components $T_{1}, \ldots, T_{n}$, each of them containing a plaquette in $\mathscr{A}$. It is clear that $n \leqq N_{A}$ because the sets $T_{i}$ are pairwise not coconnecting. Allowing empty sets $T_{i}$ we may assume $n=N_{A}$, and as in the proof of 
(3.6) in Sect. 3 we get

$$
\begin{aligned}
\sum_{\gamma_{A}}^{\prime}\left|z\left(\gamma_{A}\right)\right| & \leqq \sum_{\substack{s_{1}, \ldots, s_{n} \geqq 0 \\
\Sigma s_{i}=s}} \varepsilon^{s} \\
& =\left(\begin{array}{c}
n+s-1 \\
s
\end{array}\right) \varepsilon^{s}=\left(\prod_{i=1}^{n-1} \frac{s+i}{i}\right) \varepsilon^{s} \\
& \leqq(s+1)^{n-1} \varepsilon^{s}=(s+1)^{N_{A}-1} \varepsilon^{s}
\end{aligned}
$$

\section{Lemma A.5.}

$$
\begin{aligned}
& \sum_{m=1}^{\infty} \sum_{\gamma_{1}, \ldots, \gamma_{m}} \frac{\left|a\left(\left\{\gamma_{i}\right\}\right)\right|}{m !} \prod_{i=1}^{m}\left|z_{A}\left(\gamma_{i}\right)\right| \\
& \quad \leqq\|A\| N_{A} !\left(\frac{e}{1-e \varepsilon}\right)^{N_{A}}\left(1-\frac{8 r_{0} \varepsilon^{r_{0}}}{(1-\varepsilon)^{2}}\right)^{-1}
\end{aligned}
$$

where the sum $\sum^{\prime}$ goes over $\gamma_{1}, \ldots, \gamma_{m}$ containing exactly one polymer corresponding to $A,\|\cdot\|$ is the supremum norm.

Proof. We proceed as in the proof of Lemma A.3, but use Lemma A.4 instead of Lemma A.1. Since the minimal polymer size is 0 for polymers corresponding to $A$ we get

$$
\begin{gathered}
\omega\left(T_{n}\right) \leqq n\|A\| \sum_{s_{1} \geqq 0}\left(s_{1}+N_{A}\right)^{d_{1}}\left(1+s_{1}\right)^{N_{A}-1} \varepsilon^{s_{1}} \\
\sum_{s_{2}, \ldots, s_{n} \geqq r_{0}} \prod_{i=2}^{n} s_{i}^{d_{i}-1} \varepsilon^{s_{i}} .
\end{gathered}
$$

We now bound $\left(s_{1}+N_{A}\right)^{d_{1}}$ by $d_{1} ! \exp \left(s_{1}+N_{A}\right)$, apply Lemma 3.1 and proceed as before to obtain (A.5).

Clearly Lemma A.5 implies absolute convergence for the cluster expansions (4.5), (4.6) uniform in $\Lambda$ and $E$ provided $1 / \varepsilon>2.93(d \geqq 3)$.

Acknowledgement. I thank Erhard Seiler for helpful discussions and critical remarks concerning a first version of this paper. Especially I am indebted to him for introducing me to the method of cluster expansions (which are technically very important for this paper) and for encouraging me to study [3] and to look for a generalization for gauge theories.

\section{References}

1. Aizenman, M.: Translation invariance and instability of phase coexistence in the two dimensional Ising system. Commun. Math. Phys. 73, 83 (1980)

2. Higuchi, Y.: In: Proc. Colloquium on Random Fields (Esztergom, 1979)

3. Dobrushin, R.L.: Gibbs state describing coexistence of phases for a three dimensional Ising model. Theor. Prob. Appl. 17, 582 (1972)

4. van Beijeren, H.: Interface sharpness in the Ising system. Commun. Math. Phys. 40, 1 (1975)

5. Seiler, E.: Gauge theories as a problem of constructive quantum field theory and statistical mechanics. In: Lecture Notes in Physics, Vol. 159. Berlin, Heidelberg, New York: Springer 1982 
6. Gallavotti, G., Martin-Löf, A., Miracle-Solé, S.: Some problems connected with the description of coexisting phases at low temperatures in the Ising model. In: Statistical Mechanics and Mathematical Problems (Battell, Seattle, 1971 Rencontres), A. Lenard, ed. Lecture Notes in Physics, Vol. 20. Berlin, Heidelberg, New York: Springer 1973

7. Wegner, F.: Duality in generalized Ising models and phase transitions without local order parameters. J. Math. Phys. 12, 2259 (1971)

8. Wilson, K.: Confinement of quarks. Phys. Rev. D 10, 2445 (1974)

9. Osterwalder, K., Seiler, E.: Gauge field theories on a lattice. Ann. Phys. 110, 440 (1978)

10. Dobrushin, P.L.: The description of a Random field by means of conditional probabilities and conditions of its regularity. Theor. Prob. Appl. 13, 197 (1968)

11. Lanford, O., Ruelle, D.: Observables at infinity and states with short range correlations in statistical mechanics. Commun. Math. Phys. 13, 194 (1969)

12. Rota, G.C.: On the foundations of combinatorical theory. I. Möbius functions. Z. Wahrscheinlichkeitstheorie 2, 340 (1964)

13. Cammarota, C.: Decay of correlations for infinite range interactions in unbounded spin systems. Commun. Math. Phys. 85, 517 (1982)

14. 't Hooft, G.: Which topological features of a gauge theory can be responsible for permanent confinement? In: Recent developments in gauge theories, 't Hooft, G. et al. (ed.). New York: Plenum Press 1980

15. Borgs, C., Seiler, E.: Lattice Yang-Mills theory at non-zero temperature and the confinement problem. Commun. Math. Phys. 91,329 (1983)

16. Mack, G., Petkova, V.B.: Comparison of lattice gauge theories with gauge groups $Z_{2}$ and SU(2). Ann. Phys. (N.Y.) 123, 442 (1979)

Communicated by G. Mack

Received June 4, 1984; in revised form August 1, 1984

Note added in proof. Lemma A.5 is a very crude estimate. If one postpones the summation over the polymers corresponding to $A$ one easily gets the bound

$$
\left(1-\frac{8 r_{0} \varepsilon^{r_{0}}}{(1-\varepsilon)^{2}}\right)^{-1} \sum_{d_{1} \geqq 1} \sum_{\gamma_{A}}\left|z\left(\gamma_{A}\right)\right| \frac{1}{d_{1} !}\left[\frac{\left|\gamma_{A}\right|+N_{A}}{2 r_{0}}\right]^{d_{1}}
$$

which can e.g. be estimated by

$$
\|A\| e^{N_{A}}\left(1-\frac{8 r_{0} \varepsilon^{r_{0}}}{(1-\varepsilon)^{2}}\right)^{-1}
$$

as long as $8 r_{0} \varepsilon^{r_{0}}<(1-\varepsilon)^{2}<1$. 